\title{
Unified Models for Multiphase Coupled Inductors
}

\author{
Minjie Chen, Senior Member, IEEE, Charles R. Sullivan, Fellow, IEEE
}

\begin{abstract}
Circuit models for multiphase coupled inductors are summarized, compared, and unified. Multiwinding magnetic structures are classified into parallel-coupled structures and series-coupled structures. For parallel-coupled structures used for multiphase inductors, the relationships between a) inductance matrix models, b) extended cantilever models, c) magentic circuit models, d) multiwinding transformer models, e) gyrator-capacitor models, and f) inductance dual models are investigated and discussed. These models represent identical physical relationships in the multiphase coupled inductors, but emphasize different physical aspects and offer distinct design insights. The circuit duality between the series coupled structure and the parallel coupled structure is revealed. Design equations linking these models are compared and unified. The models and design equations are verified through theoretical derivation, SPICE simulation, and experimental measurements.
\end{abstract}

Index Terms-lumped circuit model, coupled inductor, multiphase buck converter, inductance matrix, magnetic circuit model, multi-winding transformer model, inductance dual model.

\section{INTRODUCTION}

$\mathbf{M}$ ULTIPHASE coupled inductors are widely used in many power electronics applications. Using multiphase coupled inductors in power converters can improve the efficiency, enhance the functionality, reduce the passive component size, avoid saturation, and improve the transient response [1]-[6]. Designing high performance power converters with multiphase coupled inductors need advanced models and tools.

There are two common ways of modeling multiphase coupled inductors: 1) Math-based models, which focus on the mathematical coupling relationships between windings. Mathbased models are derived based on the inductance matrix, reluctance matrix, or permeance matrix. Information about the core geometry, material properties, and winding structure is not explicitly included. Math-based models can be represented by a few convenient circuit models whose element values may not have explicit physical meanings, such as the classic $T$ model, $\pi$ model, star model, delta model, and extended cantilever model [7]-[11]. The element values of math-based models may be obtained by many different approaches, including finite-element modeling or experimental measurements. 2) Physics-based models represent the physical geometry of the magnetic structure more directly. Each portion of the magnetic structure is represented by a lumped circuit element, and many lumped circuit elements are combined into a complete circuit. The reluctance circuit model, the gyrator-capacitor model, the inductance dual model, and the modular multi-layer model

Minjie Chen is with the Department of Electrical Engineering and Andlinger Center for Energy and the Environment at Princeton University, Princeton, NJ 08540, USA.

Charles R. Sullivan is with the Thayer School of Engineering of Dartmouth College, Hanover, NH 03755, USA.

This work was jointly supported by the National Science Foundation (Award $\# 1847365$ ) and the Princeton University SEAS Innovation Fund.
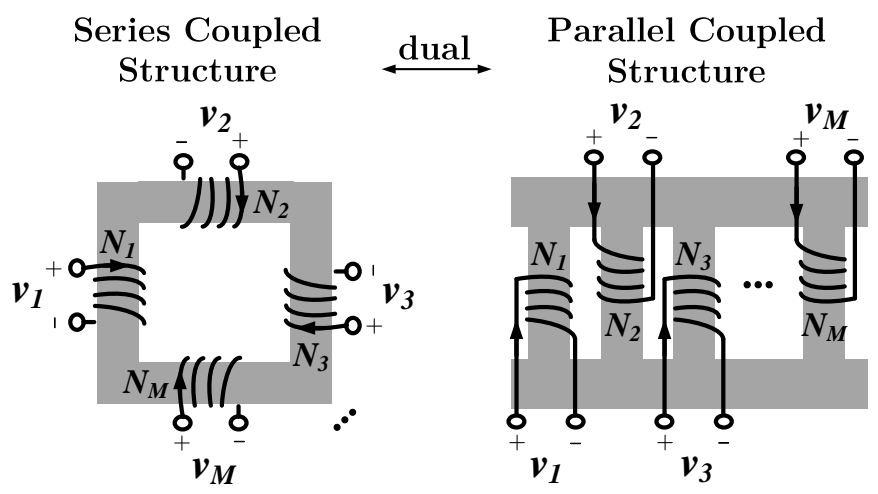

Fig. 1. Two major categories of multiphase coupled inductor structures: 1) series coupled structure; and 2) parallel coupled structure [22].

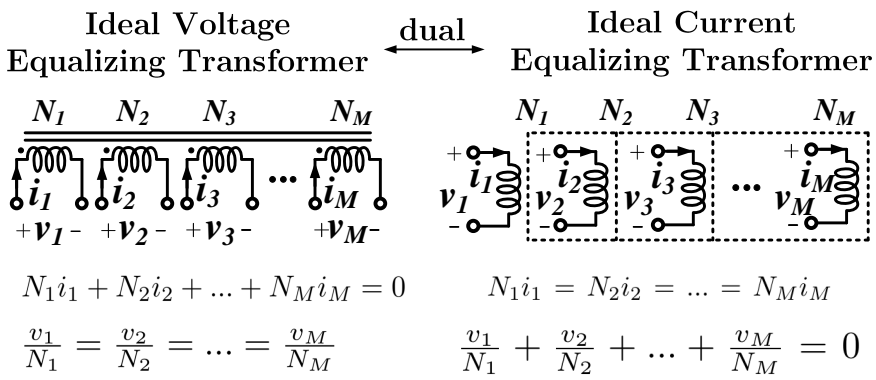

Fig. 2. Symbols and equations for: 1) ideal voltage equalizing transformer; and 2) ideal current equalizing transformer. With infinite core permeability and negligible leakage, a series coupled structure can be modeled as an ideal voltage equalizing transformer, and a parallel coupled structure can be modeled as an ideal current equalizing transformer. For the ideal current equalizing transformer, we show the core topology with dashed lines, and indicate winding polarity with arrows rather than dots, indicating the direction of current that is equal in the corresponding current equation, just as the dots indicate the direction of voltage that is equal in the corresponding voltage equation for the voltage equalizing transformer.

are physics-based models [12]-[22]. Finite-element models are physics-based models.

In their basic, linear form, all models discussed in this paper are equivalent. However, different models offer different design insights. Math-based models are well suited to theoretical analysis of power converters, especially if the magnetic components are already designed. The main advantage of physics-based models is the natural extension to capture core loss, saturation, and the details of the flux distribution in the core. Designers should choose an appropriate model based on the information that is needed in the design process. Often, moving from math-based models to physics-based models can give experienced circuit designers useful insight on circuit operation that is harder to get from math-based models.

Fig. 1 shows two major categories of multiwinding magnetic structures: 1) Series Coupled Structures, in which the flux 
paths of multiple windings are configured in series; 2) Parallel Coupled Structures, in which the flux paths of multiple windings are configured in parallel [22]. Fig. 2 shows their circuit symbols. With zero leakage flux, the series coupled structure forces the magnetic flux $\Phi$ of all windings to be the same, leading to a bonded voltage relationship across windings. With infinite permeability, the parallel coupled structure forces the magneto-motive-force (MMF) of all windings to be the same, leading to a bonded current relationship across windings. The series-coupled structure functions as an ideal voltage equalizing transformer in which $N_{1} i_{1}+N_{2} i_{2}+\ldots+N_{M} i_{M}=0$, and $\frac{v_{1}}{N_{1}}=\frac{v_{2}}{N_{2}}=\ldots=\frac{v_{M}}{N_{M}}$; the parallel coupled structure functions as an ideal current equalizing transformer in which $N_{1} i_{1}=N_{2} i_{2}=\ldots=N_{M} i_{M}$, and $\frac{v_{1}}{N_{1}}+\frac{v_{2}}{N_{2}}+\ldots+\frac{v_{M}}{N_{M}}=0$.

The goal of this paper is to investigate theoretical frameworks that are most practical for modeling sophisticated coupled magnetic structures. The series coupled structure is well studied in the literature [23]-[25]. Its mathematical symbol, an ideal voltage equalizing transformer, is commonly supported by commercial SPICE simulation platforms. The parallel coupled structure is less commonly found in power electronics applications. Moreover, ideal multi-winding current equalizing transformers are not well supported in mainstream SPICE simulation platforms. A majority of this paper focuses on investigating models for parallel coupled structures, and shows how to use these models to analyze and simulate buck converters with multiphase coupled inductors.

The remainder of this paper is organized as follows: Section II introduces general models for arbitrary multiwinding structures. Section III simplifies these models for idealized multiwinding coupled inductors. Section IV presents models for a ladder core coupled inductors and discusses the relationship between this model and a ladder modelf for a layered multiwinding transformer. Section V unifies these models and develops calculations to convert parameters between them. Section VI applies the models to symmetric coupled inductors in multiphase buck converters. Section VII verifies the effectiveness of these models, and presents simulation and experimental results of a multiphase coupled inductor buck converter. Finally, Section VIII concludes this paper.

\section{Generalized Multiwinding Structures}

The voltage and current of an arbitrary multiphase coupled inductor is described by an inductance matrix $\mathbf{L}$ :

$$
\underbrace{\left[\begin{array}{c}
v_{1} \\
v_{2} \\
\vdots \\
v_{M}
\end{array}\right]}_{\mathbf{V}}=\underbrace{\left[\begin{array}{cccc}
L_{11} & L_{12} & \cdots & L_{1 M} \\
L_{21} & L_{22} & \cdots & L_{2 M} \\
\vdots & \vdots & \ddots & \vdots \\
L_{M 1} & L_{M 2} & \cdots & L_{M M}
\end{array}\right]}_{\mathbf{L}} \underbrace{\left[\begin{array}{c}
\frac{d i_{1}}{d t} \\
\frac{d i_{2}}{d t} \\
\vdots \\
\frac{d i_{M}}{d t}
\end{array}\right]}_{\frac{d \mathbf{I}}{d t}} .
$$

Here $\mathbf{V}$ and $\mathbf{I}$ are the voltage and current of the $M$ windings. The inductance matrix is a symmetric matrix with positive and negative element values. It describes the mathematical coupling relationship between windings and is applicable to all multiwinding coupled magnetics. The element values of this matrix can be identified by finite-element modeling or

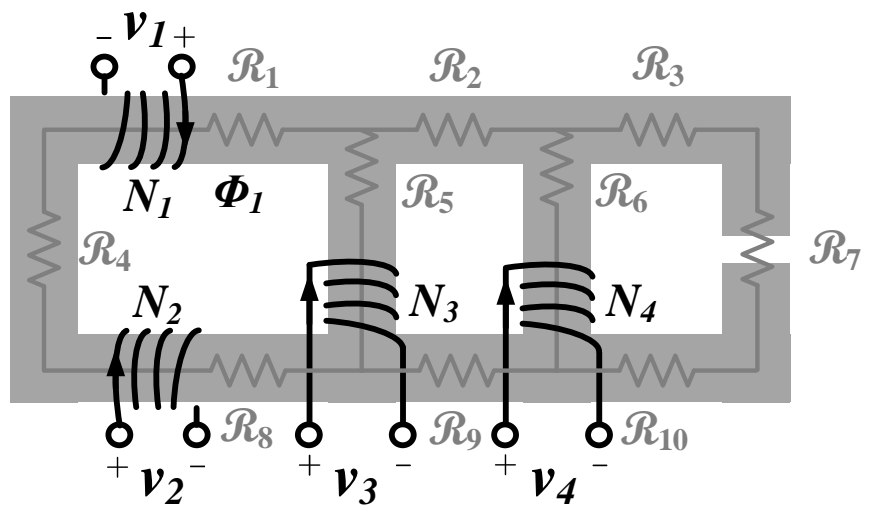

Fig. 3. An example four-winding coupled inductor with a combination of series, parallel, and air-gap configurations.

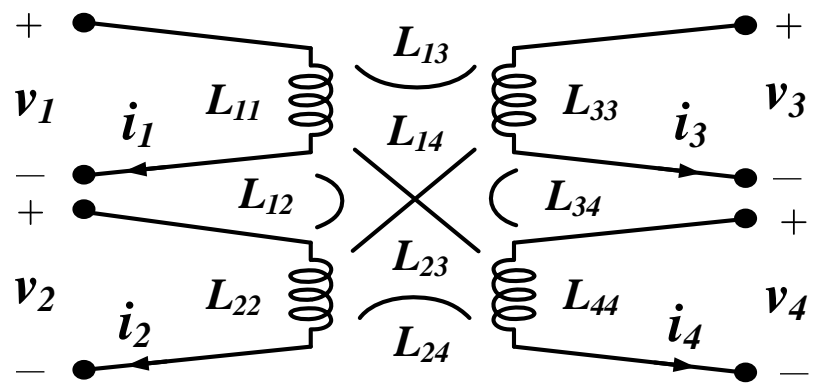

Fig. 4. Inductance matrix model of the coupled inductor in Fig. 3.

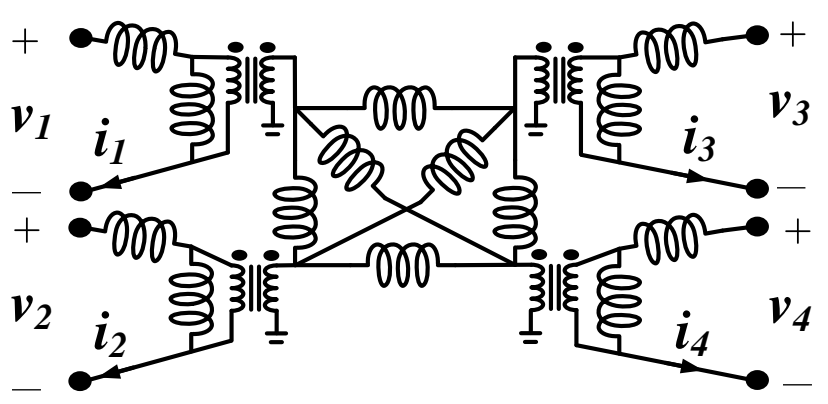

Fig. 5. Extended cantilever model of the coupled inductor in Fig. 3.

experimental measurements. Fig. 3 shows an example fourwinding coupled inductor with a combination of series, parallel, and air-gap configurations. Fig. 4 shows the inductance matrix model of this example [7]-[9]. Here the self and mutual inductance values are the element values of the inductance matrix. Many SPICE simulation platforms, e.g., PSIM, LTSpice and Simplis, support the use of the inductance matrix model. A similar model which decouples the mutual coupling relationships is the extended cantilver model [10] as shown in Fig. 5. In an extended cantilever model, all elements are non-coupled inductors and ideal transformers.

Both the inductance matrix model and the extended cantilever model are math-based models. One drawback of mathbased models is that the geometry and material property information is not explicitly shown in the model. Limited insight on magnetic structure design is offered. Physics-based models 


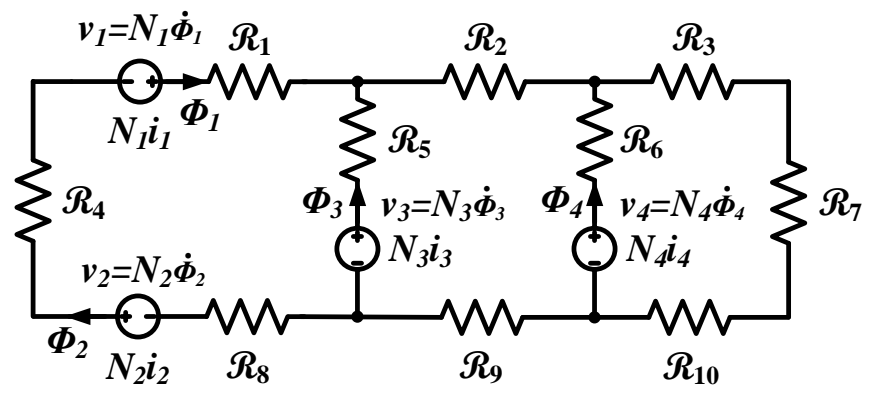

Fig. 6. Magnetic circuit model of the coupled inductor in Fig. 3.

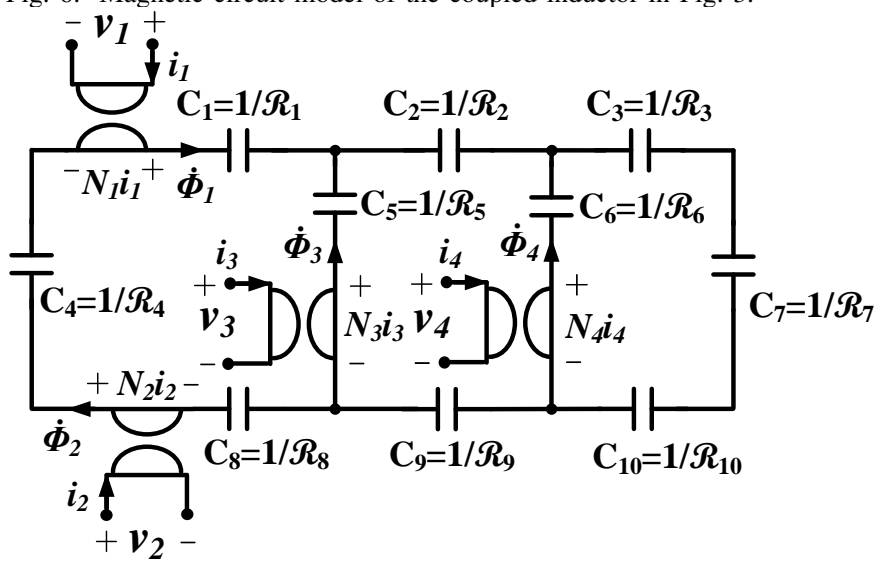

Fig. 7. Gyrator-capacitor model of the coupled inductor in Fig. 3.

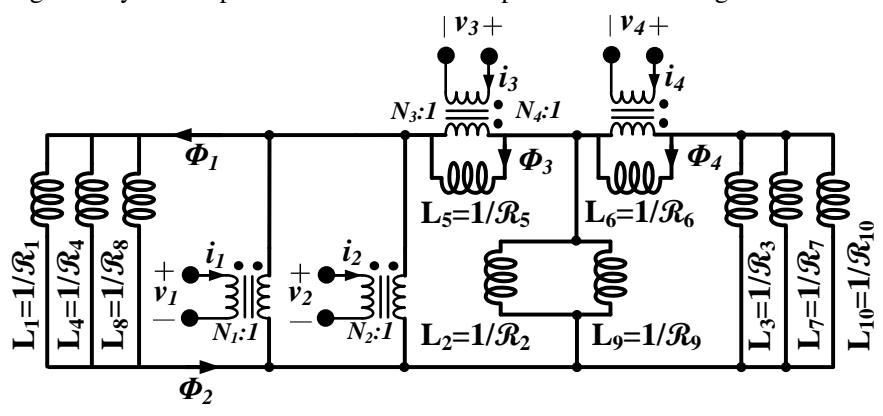

Fig. 8. Inductance dual model of the coupled inductor in Fig. 3.

can offer more direct insight on the relationship between the physical structure and the model, and are considered next.

The magnetic circuit model (reluctance circuit model) [11] [13] as shown in Fig. 6 is one of the most widely used physicsbased models. Each portion of the magnetic core is modeled as a reluctance. Each winding is modeled as an MMF source driving the reluctance circuit as a voltage source. The throughvariable is the magnetic flux $\Phi$, and the across variable is the MMF, F.

One limitation of the magnetic circuit model is that it cannot be easily simulated in SPICE. In a SPICE model, the across and through variables need to be current and voltage, instead of MMF and flux. The current is linearly related to MMF $(\mathcal{F})$, and the voltage to the derivative of flux $(d \Phi / d t)$. Added circuits are needed to implement the linear scaling and timederivative/integral relationships [14].

The gyrator-capacitor model differs from the magnetic circuit model by replacing the through variable $\Phi$ with its time- derivative $\dot{\Phi}=d \Phi / d t$, replacing the reluctances with capacitors whose capacitance equal to the permeance $\mathcal{P}=1 / \mathcal{R}$, and replacing the MMF sources with gyrators which convert current into voltage [15], [16]. Fig. 7 shows an example gyrator-capacitor model derived from the magnetic circuit model in Fig. 6. The two lumped circuit models share the same net structure but have different component values.

In the gyrator-capacitor model, the through variable has units of $\mathrm{V}$, and the across variable has units of $\mathrm{A}$. It is sometimes beneficial to apply topological duality [29]-[32] to the gyrator-capacitor model and create an inductance dual model [17]-[22], as illustrated in Fig. 8. In the inductance dual model, the elements representing the magnetic core sections are inductors with inductance values equal to the permeance values $\mathcal{P}=1 / \mathcal{R}$, in units of henry $(\mathrm{H})$. The through variable is current, and the across variable is voltage. The terminals of the model are ideal transformers with turns ratios equal to the physical turns of the windings.

All models represent the same math and physics. All models have strengths and weaknesses. The main purpose of this paper is to unify the mathematical derivations and physical insights behind these models, and systematically show their similarities, connections, and differences.

\section{IDEALIZED MUltiphase COUPLED INDUCTORS}

Fig. 9 shows an example symmetric multiwinding coupled inductor with $M$ legs on the side and one leg in the center. This is an example of a parallel coupled multiwinding structure with a symmetric geometry. Each of the outer legs is encircled by an $N$-turn winding. High permeability magnetic materials are used, and a gap is used in the center leg to control the inductance and avoid saturation with balanced dc currents. Optional small gaps are sometimes used in the outer legs to avoid saturation with small imbalances in the dc currents.

Fig. 10 shows the magnetic circuit model [12], [13] of this parallel structure. The model comprises $M$ outer leg reluctances $\mathcal{R}_{L}$, and one center leg reluctance $\mathcal{R}_{C}$. Each outer leg is driven by an MMF source $\mathcal{F}=N i$. Fig. 11 shows the gyrator-capacitor model [15], [16] of this structure, following the same changes in variables as with Figs. 6 and 7.

Finding the topological dual of the magnetic circuit or the gyrator-capacitor model results in the inductance dual model shown in Fig. 12. To capture the dc bias of the magnetic core in circuit simulations, the ideal transformers in the inductance dual model should operate in dc, i.e., the transformer current relationship should apply to dc as well as ac currents. However, this is insufficient to make the the model in Fig. 12 capture the dc bias level in each core path, because there is a loop of ideal inductors for which the dc current is undetermined. To solve this problem, the model in Fig. 13 includes a small, non-physical resistance in series with each inductor $\left(r_{L}\right)$. For proper dc balancing, the resistance of each is proportional to, but orders of magnitude smaller than, the impedance of the inductor it is connected in series with, divided by the quality factor of the core material at this frequency, to ensure that the losses in this dc balancing resistor are negligible $\left(r_{L}=\right.$ $\left.\varepsilon / \mathcal{R}_{L}, r_{C}=\varepsilon / \mathcal{R}_{C}, \varepsilon \ll \omega_{S} / Q\right)$, where $\omega_{S}$ is the switching 


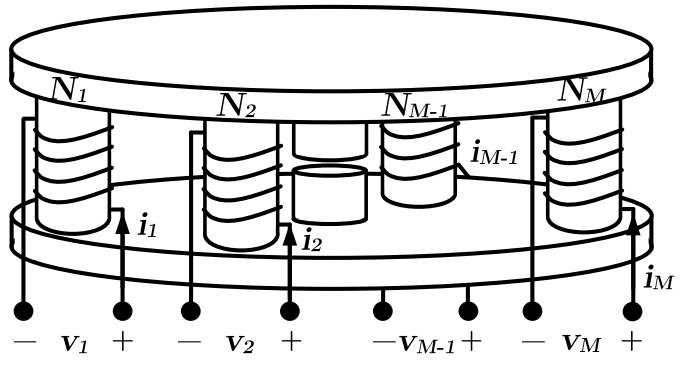

Fig. 9. A symmetric multiphase coupled inductor with many windings. This structure has $M$ side legs and a center leg.

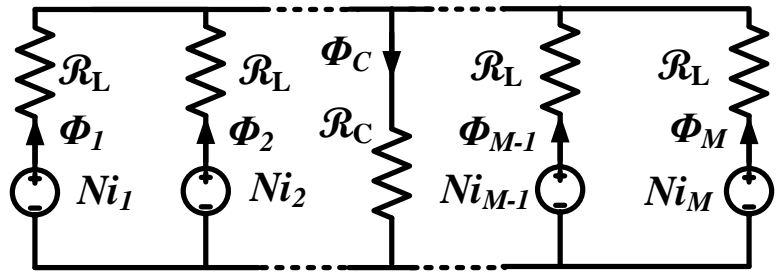

Fig. 10. Magnetic circuit model of the structure in Fig. 9. All windings have $N$ turns. The reluctances of the side legs are $\mathcal{R}_{L}$, and the reluctance of the center leg is $\mathcal{R}_{C}$. This structure is fully symmetric.

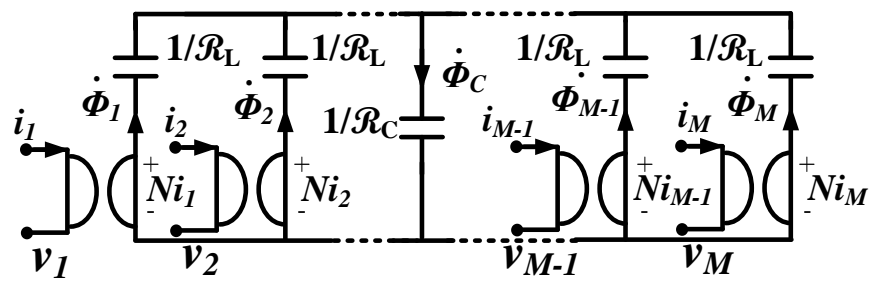

Fig. 11. Gyrator-capacitor model of the structure in Fig. 9. All windings have $N$ turns. Labeled values are capacitance values. The across variable is $\mathcal{F}$; the through variable is $\dot{\Phi}=d \Phi / d t$.

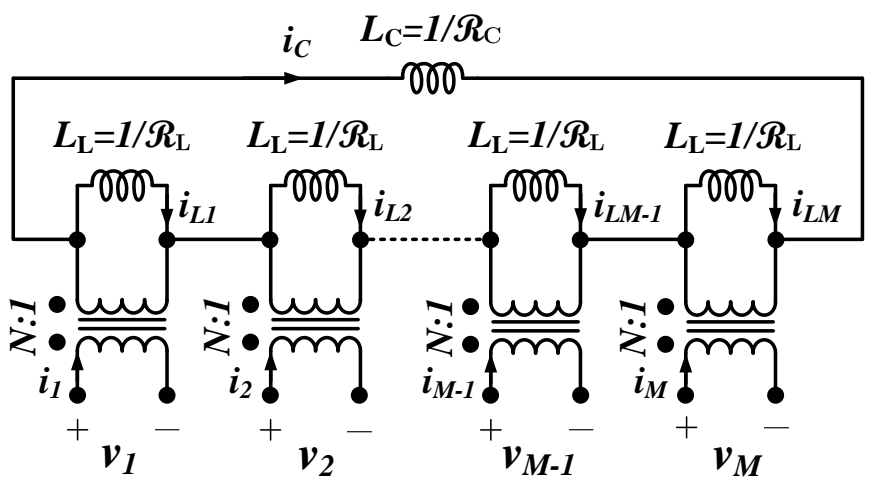

Fig. 12. Inductance dual model of the structure in Fig. 9. $1 / \mathcal{R}_{C}$ and $1 / \mathcal{R}_{L}$ represent the inductive elements of the center leg and the side legs, respectively. In SPICE simulations with ideal transformers, $i_{C}$ and $i_{L}$ are linearly related to $\Phi_{C}$ and $\Phi_{L}$ in the center leg and the side legs, respectively.

frequency and $Q$ is the quality factor of the core material at this frequency. With the flux, including dc components, represented by inductor currents, one can probe the currents $i_{L}(t)$ and $i_{C}(t)$, divide them by the corresponding reluctances $\mathcal{R}_{L}$ and $\mathcal{R}_{C}$, and visualize the magnetic fluxes $\Phi_{L}(t)$ and $\Phi_{C}(t)$ in circuit simulations. Fig. 13 also shows one example way to

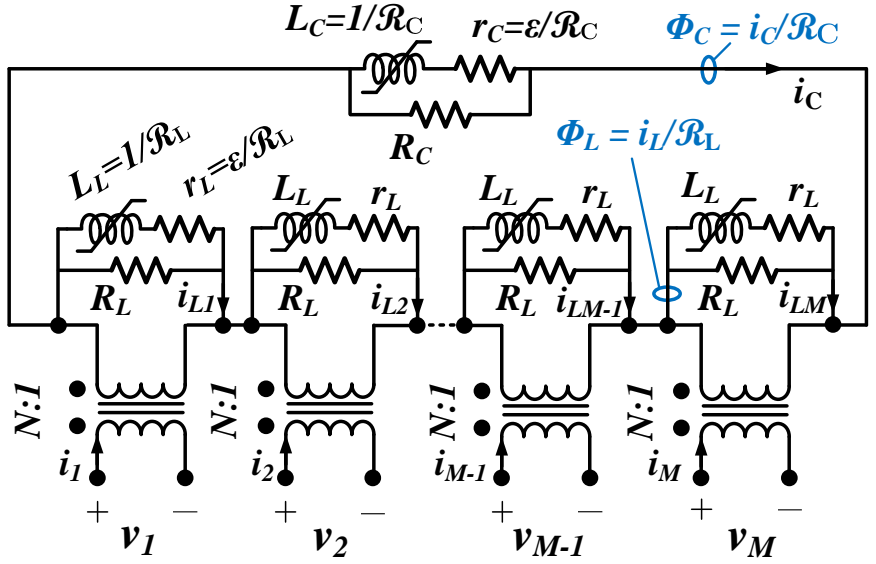

Fig. 13. Inductance dual model including saturable inductors and core-loss resistors. The series resistor $r_{L}$ and $r_{C}$ determines the dc current. The parallel resistors $R_{L}$ and $R_{C}$ capture the core loss. By probing the current in the inductance dual model and divide it with the reluctance value, the magnetic flux and saturation effects in each portion of the core can be visualized.

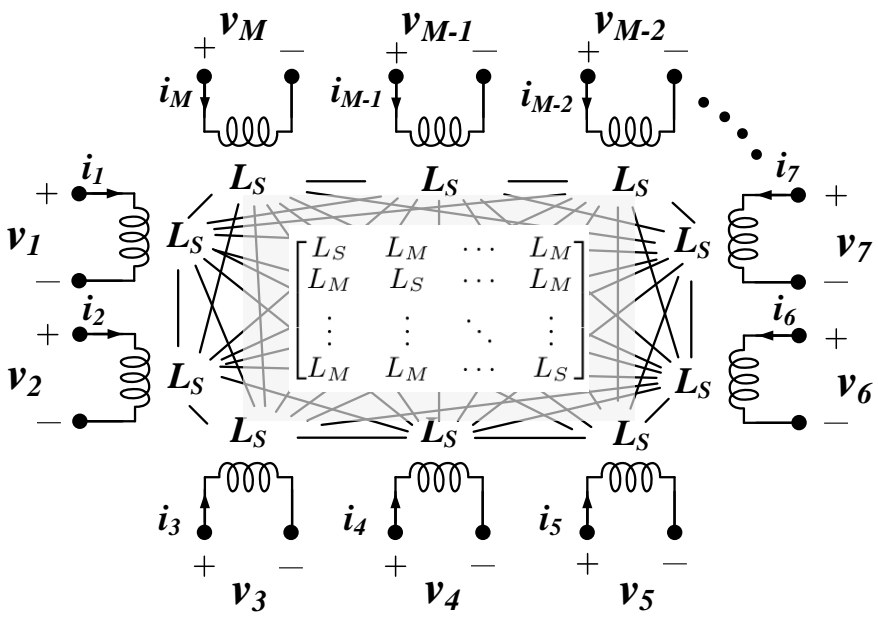

Fig. 14. Inductance matrix model of the structure in Fig. 9. The element values of this model come from the inductance matrix.

implement core loss and saturation effects in the inductance dual model. Each portion of the magnetic core is implemented as a saturable inductor $\left(L_{L}\right.$ or $\left.L_{C}\right)$. An additional resistor is then connected in parallel with the saturable inductor and resistor to capture the core loss of each portion of the magnetic core.

Fig. 14 shows the inductance matrix model of a multiwinding coupled inductor. The core of this model is a $M \times M$ inductance matrix which represents the mathematical mapping relationships between windings. The inductance matrix model is interchangeable with a multiwinding transformer model, as well as with all the other models under discussion. There are many ways to implement a multiwinding transformer model. Fig. 15 shows one example implementation of the multiwinding transformer model using an ideal current equalizing transformer [6]. The magnetizing inductance of each winding is $\frac{M}{M-1} L_{\mu}$ and the leakage inductance of each winding is $L_{l}$. The self inductance $L_{S}$ of this model in the indutance matrix equation is $L_{\mu}+L_{l}$. The mutual inductance $L_{M}$ is $-\frac{1}{M-1} L_{\mu}$. 


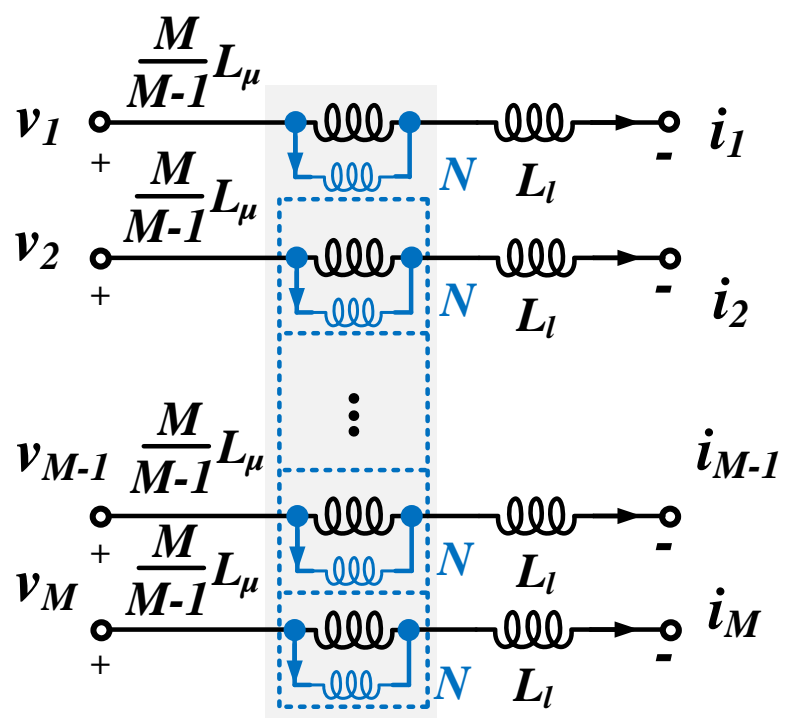

Fig. 15. Multiwinding transformer model of the structure in Fig. 9 implemented with an ideal current equalizing transformer (in blue). The ideal current equalizing transformer equalizes the MMFs of all blue windings and forces the summations of the volt-per-turn of all windings to be zero.

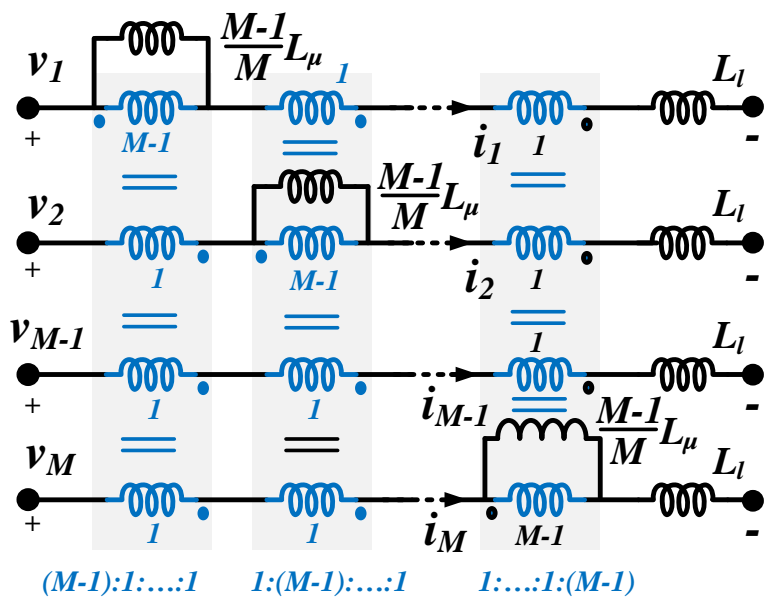

Fig. 16. Multiwinding transformer model of the structure in Fig. 9 implemented with an ideal voltage equalizing transformer (in blue). The ideal voltage equalizing transformer equalizes the volts-per-turn of all blue windings and forces the summation of MMF of all windings to be zero.

The turns ratio of is $\{1: 1: \ldots: 1\}$, assuming equal numbers of turns $N$ in each physical winding.

Fig. 16 shows another implementation of a multiwinding transformer model using $M$ ideal voltage equalizing transformers. Each of these transformers has a turns ratio of $\{(M-1): 1: \ldots: 1\}$ (assuming all physical windings have the same number of turns). The magnetizing inductance reflected on the $\{M-1\}$ turn side is $\frac{M-1}{M} L_{\mu}$. The leakage inductance of each winding is $L_{l}$. More discussion of multiwinding transformer models is provided in [33]-[39].

The inductance matrix model, the extended cantilever model, and the multiwinding transformer models are mathbased models. The magnetic circuit model, the gyratorcapacitor model, and the inductance dual model are physicsbased models. Math-based models describe the mathematical equations at the interface, from port-to-port. Physics-based models illustrate the physical behaviors of the magnetic core and the windings.

\section{LAdDer Core and Layered Winding Structures}

In this section, we first consider a commonly used coupled inductor structure, the ladder core, and relax the assumption that the top and bottom plates have negligible reluctance to develop a more accurate model. Then we consider a conventional multi-winding transformer with layered windings, to illustrate the topological dual relationship between this structure and the ladder-core structure.

\section{A. Ladder Core Structure}

Fig. 17 shows a parallel coupled structure implemented with a ladder core [40]-[45]. This ladder core structure is attractive in designing coupled inductors for multiphase buck converters. If the reluctance of the top and bottom bars of this structure are neglected, any of the models previously discussed apply to this structure. But unless they are very thick and/or have very high permeability, their reluctance may be significant, and the magnetic circuit model in Fig. 18 applies. Although there is no explicit leakage path shown in Fig. 17, the flux leakage between the top and bottom bar is important to the operation of this structure in a typical circuit, and is modeled by the four leakage reluctances, $\mathcal{R}_{l}$. Figs. 19-20 show the corresponding gyrator-capacitor and inductance dual models, respectively. Based on Fig. 18, the magnetic fluxes $\left[\Phi_{1}, \ldots\right.$, $\left.\Phi_{M}\right]^{T}$ are functions of $\left[\mathcal{F}_{1}, \ldots, \mathcal{F}_{M}\right]^{T}$, which are the MMF values at the nodes labeled in Fig. 18. The magnetic fluxes and MMFs are linked by a $M \times M$ permeance matrix $\mathbf{P}$ with element values determined by reluctance $\mathcal{R}_{l}$ and $\mathcal{R}_{t}$ :

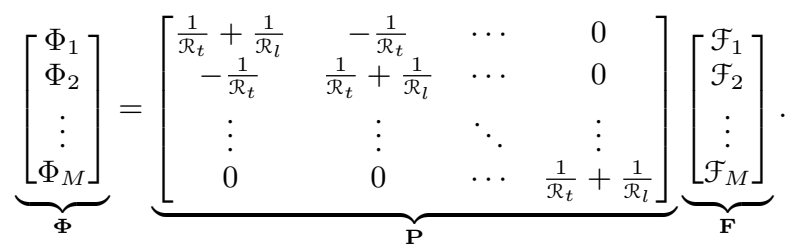

Using $\Phi_{x}=\frac{N i_{x}-\mathcal{F}_{x}}{\mathcal{R}_{\mu}}$, the vector of current in all windings (I) is a function of the vector of fluxes $(\boldsymbol{\Phi})$ in all legs and the permeance matrix $(\mathbf{P})$ :

$$
\mathbf{I}=\frac{1}{N}\left(\mathcal{R}_{\mu}+\mathbf{P}^{-1}\right) \mathbf{\Phi}
$$

Taking the derivative of (3) results in the inductance matrix relationship connecting the winding current vector (I) and the winding voltage vector $(\mathbf{V})$ :

$$
\frac{d \mathbf{I}}{d t}=\frac{1}{N^{2}}\left(\mathcal{R}_{\mu}+\mathbf{P}^{-1}\right) \mathbf{V} .
$$

\section{B. Layered Winding Structure}

In this subsection, we consider a conventional multiwinding transformer with layered windings. These may be concentric wire- or foil-wound windings or stacked PCB windings, as shown in Fig. 21. Fig. 21 also shows a general schematic of this type of structure with multiple windings coupled to a 


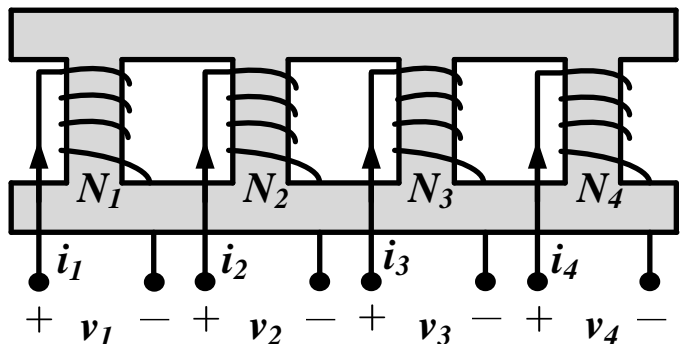

Fig. 17. Multiwinding coupled magnetics with a ladder core. Windings are parallel coupled with equalized MMF and zero-summed voltage-per-turn.

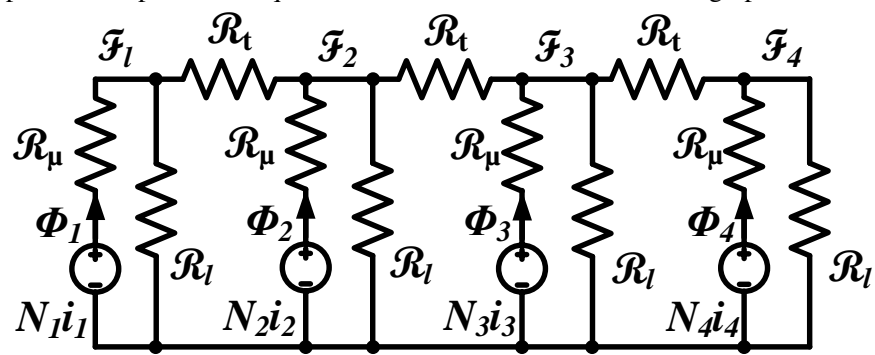

Fig. 18. Magnetic circuit model of the ladder structure in Fig. 17. Labeled values $\mathcal{R}$ are reluctance values.

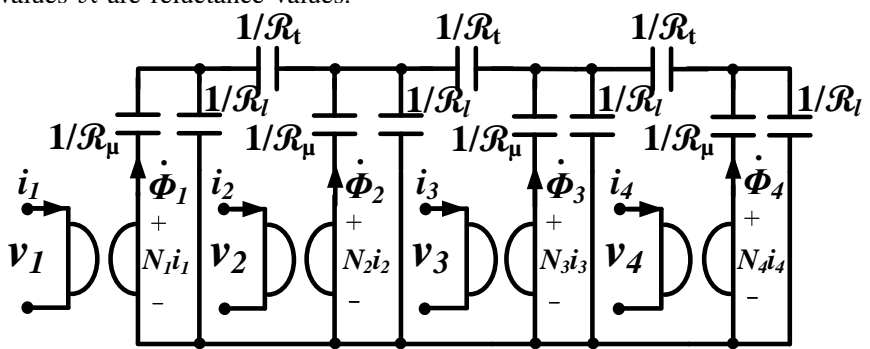

Fig. 19. Gyrator-capacitor model of the ladder structure in Fig. 17. Labeled values $1 / \mathcal{R}$ are capacitance values.

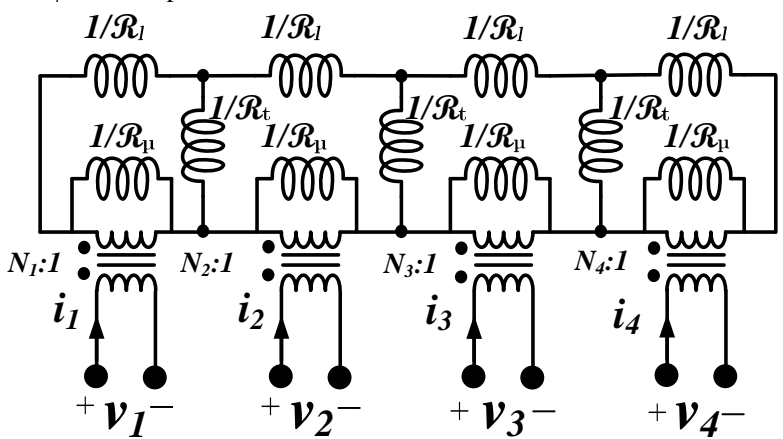

Fig. 20. Inductance dual model of the ladder structure in Fig. 17. Labeled values $1 / \mathcal{R}$ are inductance values.

single flux path. The corresponding magnetic circuit model, gyrator-capacitor model, and inductance dual model of the layered structure, including a leakage path between each winding layer represented by $\mathcal{R}_{K}$, are shown in Figs. 22-24, respectively. The inductance dual model of Fig. 24 can be further extended to cover skin and proximity effects [23]-[25].

A duality is observed between the ladder core models and the layered winding models. The ladder core structure has MMF sources in parallel, and the layered winding structure has MMF sources in series. The ladder core structure requires

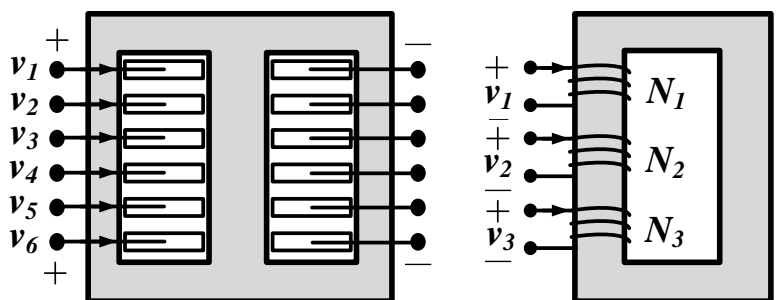

Fig. 21. Multiwinding series coupled planar magnetic structure. Windings are series coupled with equalized voltage-per-turn and zero summed MMF.

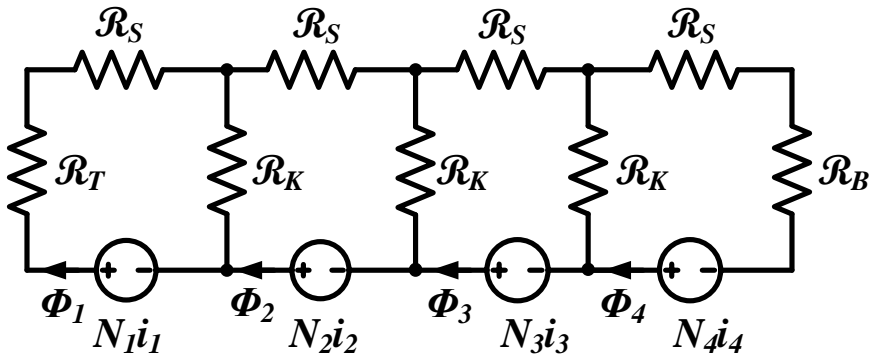

Fig. 22. Magnetic circuit model of the planar structure in Fig. 21. Labeled values are reluctance values.

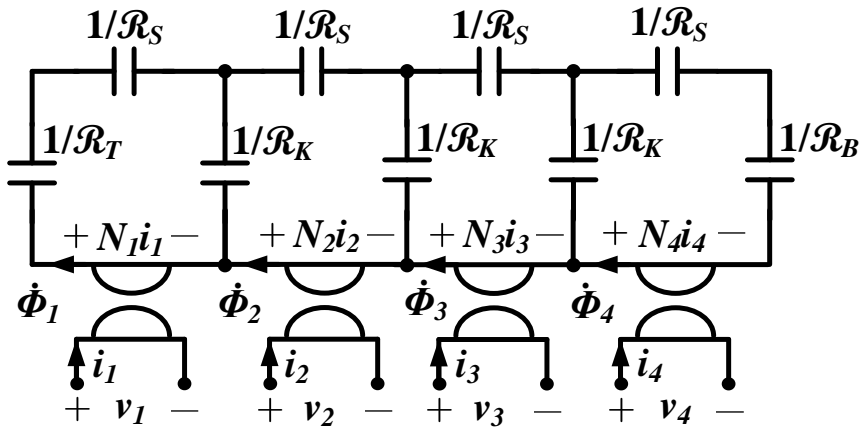

Fig. 23. Gyrator-capacitor model of the layered transformers such as the planar structure in Fig. 21. Labeled values are capacitance values.

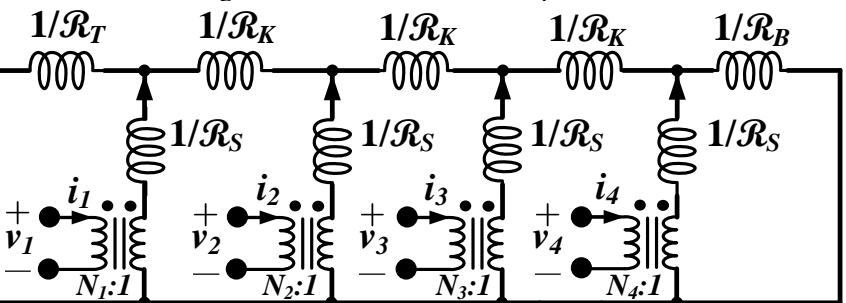

Fig. 24. Inductance dual model of the planar structure in Fig. 21. Labeled values are inductance values. The inductance dual model is very similar to the modular layer model presented in [23].

windings to have similar MMFs, and the layered winding structure requires windings to have similar fluxes. The ladder core structure is usually used to couple windings to make their ac currents match more closely, and the layered winding structure is usually used to couple windings to achieve similar voltage per turn. The ladder core structure functions well for coupled inductors in multiphase buck converters, and the layered winding structure functions well for multiport $\mathrm{dc}-\mathrm{dc}$ converters, such as multi-active-bridge (MAB) converters and energy routers [26]-[28]. 


\section{UNIFYING MOdELS FOR IDEALIZED STRUCTURES}

This section unifies and compares simplified models for coupled inductors in which the top and bottom plate or bar reluctance is neglected and the model is symmetric. The equations which describe the inductance matrix model in Fig. 14 is the inductance matrix $\mathbf{L}$ in (1). The multiwinding transformer model in Fig. 15 and Fig. 16 correspond to (1) with $L_{S}=L_{\mu}+L_{l}$ and $L_{M}=-\frac{1}{M-1} L_{\mu}$. The magnetic circuit model in Fig. 10 can be described by a reluctance matrix $\mathbf{R}$ based on $\mathcal{R}_{L}$ and $\mathcal{R}_{C}$ :

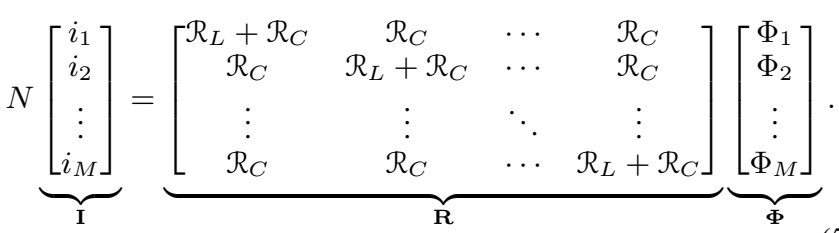

The equation which describes the gyrator-capacitor model and the inductance dual model is the time derivative of (5):

$$
N \frac{d \mathbf{I}}{d t}=\mathbf{R} \frac{d \mathbf{\Phi}}{d t}=\mathcal{R}_{L} \frac{d \mathbf{\Phi}}{d t}+\mathcal{R}_{C} \frac{d \Phi_{C}}{d t}=\frac{\mathcal{R}_{L}}{N} \mathbf{V}+\frac{\mathcal{R}_{C}}{N} \sum_{x=1}^{M} v_{x} .
$$

Note that $\mathbf{L}=N^{2} \mathbf{R}^{-1}$. As a result:

$$
\begin{aligned}
L_{S} & =\frac{N^{2}\left(\mathcal{R}_{L}+(M-1) \mathcal{R}_{C}\right)}{\mathcal{R}_{L}\left(\mathcal{R}_{L}+M \mathcal{R}_{C}\right)}=L_{\mu}+L_{l}, \\
L_{M} & =\frac{-N^{2} \mathcal{R}_{C}}{\mathcal{R}_{L}\left(\mathcal{R}_{L}+M \mathcal{R}_{C}\right)}=-\frac{1}{M-1} L_{\mu}, \\
L_{l} & =\frac{N^{2}}{\mathcal{R}_{L}+M \mathcal{R}_{C}}=L_{S}+(M-1) L_{M}, \\
L_{\mu} & =\frac{N^{2}(M-1) \mathcal{R}_{C}}{\mathcal{R}_{L}\left(\mathcal{R}_{L}+M \mathcal{R}_{C}\right)}=-(M-1) L_{M} .
\end{aligned}
$$

In a highly coupled structure, $\mathcal{R}_{L} \ll \mathcal{R}_{C}$, the relationships between $L_{S}, L_{M}, L_{\mu}, L_{l}, \mathcal{R}_{L}$ and $\mathcal{R}_{C}$ become:

$$
\begin{gathered}
L_{S} \approx \frac{(M-1) N^{2}}{M \mathcal{R}_{L}}, \\
L_{M} \approx \frac{-N^{2}}{M \mathcal{R}_{L}}, \\
L_{l} \approx \frac{N^{2}}{M \mathcal{R}_{C}}, \\
L_{\mu} \approx \frac{(M-1) N^{2}}{M \mathcal{R}_{L}} .
\end{gathered}
$$

To design a multiphase coupled inductor, it is important to be able to estimate the dc flux density in each portion of the core. In the inductance dual model, the current that goes through $L_{C}=1 / \mathcal{R}_{C}, i_{C}$, is related to $\Phi_{C}$. The current in each of the legs, $i_{L 1}, i_{L 2}, \ldots, i_{L M}$ is related to the flux going through the corresponding side leg of the core, $\Phi_{1}, \Phi_{2}, \ldots, \Phi_{M}$ :

$$
\begin{gathered}
\frac{d \Phi_{C}}{d t}=\frac{\sum_{x=1}^{M} v_{x}}{N}=\frac{1}{\mathcal{R}_{C}} \frac{d i_{C}}{d t}, \\
\frac{d \Phi_{x}}{d t}=\frac{v_{x}}{N}=\frac{1}{\mathcal{R}_{L}} \frac{d i_{L x}}{d t} .
\end{gathered}
$$

If the model in Fig. 13 is used, then

$$
\Phi_{C}=\frac{i_{C}}{\mathcal{R}_{C}} ; \Phi_{x}=\frac{i_{L x}}{\mathcal{R}_{L}} .
$$

Assuming the dc currents of all winding are all equal $I_{1}=$ $I_{2}=\ldots=I_{M}=I_{D C}$, the dc flux densities in the side legs $\left(\Phi_{L, D C}\right)$ and center leg $\left(\Phi_{C, D C}\right)$ of the magnetic core, which determines the peak flux density when the system is working at full load, are functions of $M, N, \mathcal{R}_{L}, \mathcal{R}_{C}$, and $I_{D C}$ :

$$
\begin{aligned}
& \Phi_{L, D C}=\frac{N I_{D C}}{\mathcal{R}_{L}+M \mathcal{R}_{C}}, \\
& \Phi_{C, D C}=\frac{M N I_{D C}}{\mathcal{R}_{L}+M \mathcal{R}_{C}} .
\end{aligned}
$$

\section{Multiphase Coupled Inductor Buck CONVERTER}

Coupled inductors can greatly enhance the performance of multiphase buck converters [34]-[36], [40]-[49]. By coupling the multiple inductors with a high permeability magnetic core, one can significantly reduce the current ripple in each of the phase in order to reduce the conduction loss in switches, windings, and printed circuit boards. Many methods have been developed to evaluate the performance of a coupled inductor design in multiphase buck converters. Based on the inductance matrix model, [47] predicts that the current ripple ratio perphase between the uncoupled and coupled cases with the same transient inductances in a two-phase buck converter is:

$$
\frac{\Delta i_{c p}}{\Delta i_{\text {noncp }}}=\frac{1+\frac{D}{1-D} \alpha}{1-\alpha},
$$

where $\alpha=\frac{L_{M}}{L_{S}}=-\frac{\mathcal{R}_{C}}{\mathcal{R}_{L}+\mathcal{R}_{C}}$, and $D$ is the duty ratio. This ripple ratio is an important figure-of-merit (FOM) for evaluating coupled inductor design. A smaller ripple ratio is better. Substituting $\alpha$ into Eq. (20), the ripple ratio becomes a function of $\mathcal{R}_{L}, \mathcal{R}_{C}$, and duty ratio $D$ :

$$
\frac{\Delta i_{c p}}{\Delta i_{\text {noncp }}}=\frac{(1-D) \mathcal{R}_{L}+(1-2 D) \mathcal{R}_{C}}{(1-D)\left(\mathcal{R}_{L}+2 \mathcal{R}_{C}\right)} .
$$

This equation was generalized in [43] for a $M$-phase coupled inductor buck converter with $0<D<\frac{1}{M}$ by using a multiwinding transformer model. We define $\rho$ as the ratio between $L_{\mu}$ and $L_{l}$ in the multiwinding transformer model as shown in Fig. 16:

$$
\rho=\frac{L_{\mu}}{L_{l}}=\frac{(M-1) \mathcal{R}_{C}}{\mathcal{R}_{L}} .
$$

The ripple ratio is a function of $M, D, \mathcal{R}_{L}$ and $\mathcal{R}_{C}$ :

$$
\frac{\Delta i_{c p}}{\Delta i_{\text {noncp }}}=\frac{\frac{M-1+\rho}{M-1+M \rho}-D}{1-D}=\frac{(1-D) \mathcal{R}_{L}+(1-M D) \mathcal{R}_{C}}{(1-D)\left(\mathcal{R}_{L}+M \mathcal{R}_{C}\right)} \text {. }
$$

Four design parameters were defined in [6] based on the inductance matrix model for a $M$-phase coupled inductor buck converter with an arbitrary duty ratio $D$ by defining an index $k$ to indicate the number of phases that are simultaneously energized. $D$ and $k$ are related by $\frac{k}{M}<D<\frac{k+1}{M}$ :

1) Overall steady-state inductance $\left(L_{o s s}\right)$ : the multiphase coupled inductor results in the same total output peak-topeak ripple current amplitude as a single discrete inductor with inductance $L_{o s s}$.

2) Per-phase steady-state inductance $\left(L_{p s s}\right)$ : each phase of the multiphase coupled inductor has the same peak-topeak steady-state current ripple as with individual discrete inductors with inductance $L_{p s s}$; 
3) Overall transient inductance $\left(L_{o t r}\right)$ : the multiphase coupled inductor behaves as a single discrete inductor with $L_{o t r}$ as the inductance for the purposes of evaluating the overall transient performance and small signal model;

4) Per-phase transient inductance $\left(L_{p t r}\right)$ : the same physical meaning as the overall transient inductance, but normalized on a per-phase basis by dividing the overall transient inductance by the number of phases.

These parameters are expressed as functions of $L_{S}, L_{M}$, $D, M$, and $k$ in [6] based on the inductance matrix model:

$$
\begin{aligned}
L_{o s s} & =\frac{(1-D) D M\left(L_{S}+L_{M}(M-1)\right)}{(D M-k)(1+k-D M)}, \\
L_{p s s} & =\frac{\left(L_{S}-L_{M}\right)\left(L_{S}+(M-1) L_{M}\right)}{L_{S}+\left((M-2 k-2)+\frac{k(k+1)}{M D}+\frac{M D(M-2 k-1)+k(k+1)}{M(1-D)}\right) L_{M}}, \\
L_{\text {otr }} & =\frac{L_{S}+(M-1) L_{M}}{M}, \\
L_{p t r} & =L_{S}+(M-1) L_{M} .
\end{aligned}
$$

With these design parameters, the steady-state output current ripple, the output small-signal model, per-phase current ripple, and per-phase small-signal model of the multiphase coupled inductor, working in continuous-conduction-mode, can be rapidly estimated by applying them in the standard equations for a single-phase buck converter with the same duty ratio $D$ and the same switching frequency $f$.

These effective inductance values are also derived in Appendix I based on the inductance dual model. $L_{p s s}, L_{p t r}, L_{o s s}$, and $L_{o t r}$ expressed as functions of $\mathcal{R}_{L}, \mathcal{R}_{C}, D, M$, and $k$ are:

$$
\begin{aligned}
L_{o s s} & =\underbrace{\frac{(1-D) D M}{(k+1-D M)(D M-k)}}_{1 / \delta} \times \underbrace{\frac{N^{2}}{\mathcal{R}_{L}+M \mathcal{R}_{C}}}_{L_{p t r}}, \\
L_{p s s} & =\frac{N^{2}(1-D)}{-\frac{k^{2} \mathcal{R}_{C}}{D M}-\frac{k \mathcal{R}_{C}}{D M}+2 k \mathcal{R}_{C}-D M \mathcal{R}_{C}+\mathcal{R}_{C}-D \mathcal{R}_{L}+\mathcal{R}_{L}}, \\
L_{\text {otr }} & =\frac{1}{M} \times \underbrace{\frac{N^{2}}{\mathcal{R}_{L}+M \mathcal{R}_{C}}}_{L_{p t r}}, \\
L_{p t r} & =\frac{N^{2}}{\mathcal{R}_{L}+M \mathcal{R}_{C}} .
\end{aligned}
$$

The per-phase transient inductance $L_{p t r}$ is a key parameter in the design process. It is equal to the leakage inductance $L_{l}$ in the transformer models, which show how it functions in the circuit. We define a coefficient $\delta$ such that $L_{o s s}=L_{p t r} / \delta . \delta$ also quantifies the combined output ripple reduction compared to using a single-phase converter with an inductor value $L_{p t r}$. This factor is related to the use of multiphase interleaving [50]-[52], and is not affected by coupling. Note that a singlephase converter with an inductance value $L_{p t r}$ is a questionable choice for a reference point, given that such a converter would have worse transient response than the coupled-inductor design with the same $L_{p t r}$ The overall transient inductance $L_{o t r}$ is simply $L_{p t r}$ scaled by $1 / M$; effectively $M$ discrete inductors with inductance $L_{p t r}=L_{l}$ connected in parallel, as can be seen from the transformer models.

Table I summaries the equations for calculating these design parameters based on the inductance matrix model, inductance
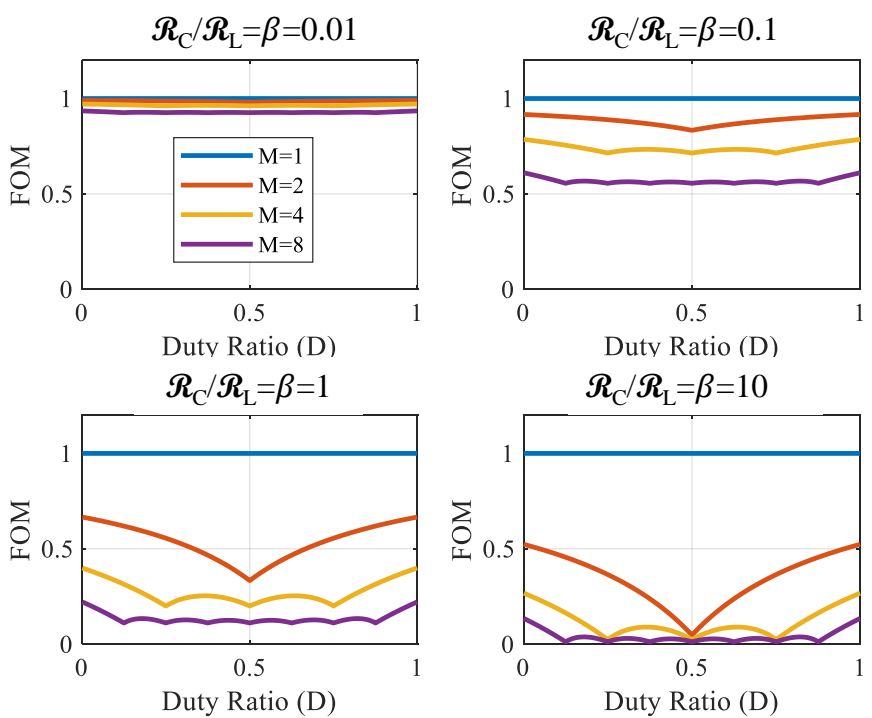

Fig. 25. FOM of the multiphase coupled buck inductor as functions of the number of phases $(M)$, duty ratio $(D)$, and reluctance ratio $\left(\beta=\mathcal{R}_{C} / \mathcal{R}_{L}\right)$. A lower FOM indicates more winding current ripple reduction. A high $\beta$ indicates strong coupling, and a low $\beta$ indicates weak coupling.

dual model, and multiwinding transformer model. A software tool - Princeton CoupL - was developed and is available at: http://www.princeton.edu/ minjie/coupL/coupL.html.

We define a Figure of Merit (FOM) as the ratio between the transient and steady-state inductance per-phase:

$$
\begin{aligned}
& \text { FOM } \stackrel{\text { def }}{=} \frac{L_{p t r}}{L_{p s s}}=\frac{\Delta i_{c p}}{\Delta i_{\text {noncp }}} \\
& =\frac{-\frac{k^{2} \mathcal{R}_{C}}{D M}-\frac{k \mathcal{R}_{C}}{D M}+2 k \mathcal{R}_{C}-D M \mathcal{R}_{C}+\mathcal{R}_{C}-D \mathcal{R}_{L}+\mathcal{R}_{L}}{(1-D)\left(\mathcal{R}_{L}+M \mathcal{R}_{C}\right)} \\
& =\frac{-\frac{k^{2} \beta}{D M}-\frac{k \beta}{D M}+2 k \beta-D M \beta+\beta-D+1}{(1-D)(1+M \beta)} .
\end{aligned}
$$

Here $\beta=\frac{\mathcal{R}_{C}}{\mathcal{R}_{L}}$. This ratio is equal to the per-phase current ripple ratio $\frac{\Delta i_{c p}}{\Delta i_{\text {noncp }}}$. Fig. 25 plots this FOM for a range of $D$, $M$, and $\beta$. TheFOM is always between zero and one. A smaller FOM is better. If $\beta \rightarrow+\infty, \mathcal{R}_{C} \gg \mathcal{R}_{L}$, the inductors are strongly coupled, the benefits of coupling increase. If $\beta \rightarrow 0$, $\mathcal{R}_{L} \ll \mathcal{R}_{C}$, the inductors are weakly coupled, and the benefits of coupling decrease.

In practice, $\beta$ can be increased by reducing $\mathcal{R}_{L}$, by using high permeability core material, reducing length of the legs, and increasing area of the legs. $\mathcal{R}_{C}$ is then adjusted to maintain the selected $L_{p t r}$ to meet the transient requirements while maintain a small ripple. Tradeoffs exist between core loss, saturation margin, energy storage requirements, and transient response. In an optimal design, the core loss, winding loss, efficiency, power density, and transient and steady-state performance are highly correlated and need to be jointly optimized for a given design specification.

A good strategy to design high performance coupled inductors for multiphase buck converter is:

1) Selected a magnetic structure with $\mathcal{R}_{L} \ll \mathcal{R}_{C}$;

2) Choose an appropriate per-phase transient inductance $\left(L_{p t r}=L_{l}\right)$ based on the tradeoff between transient 
TABLE I

UNIFIEd MOdEls For Multiphase COUPLED INDUCtors

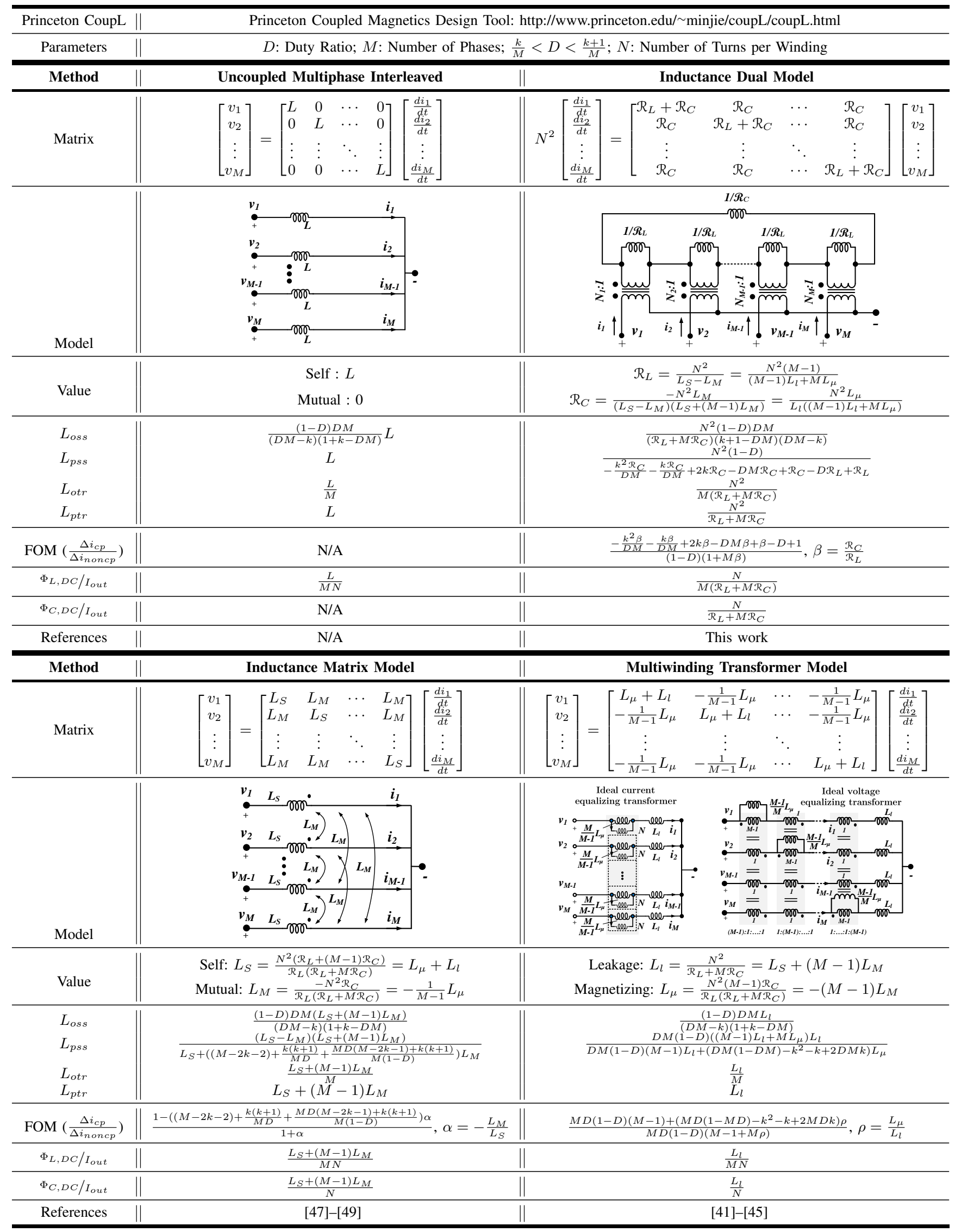



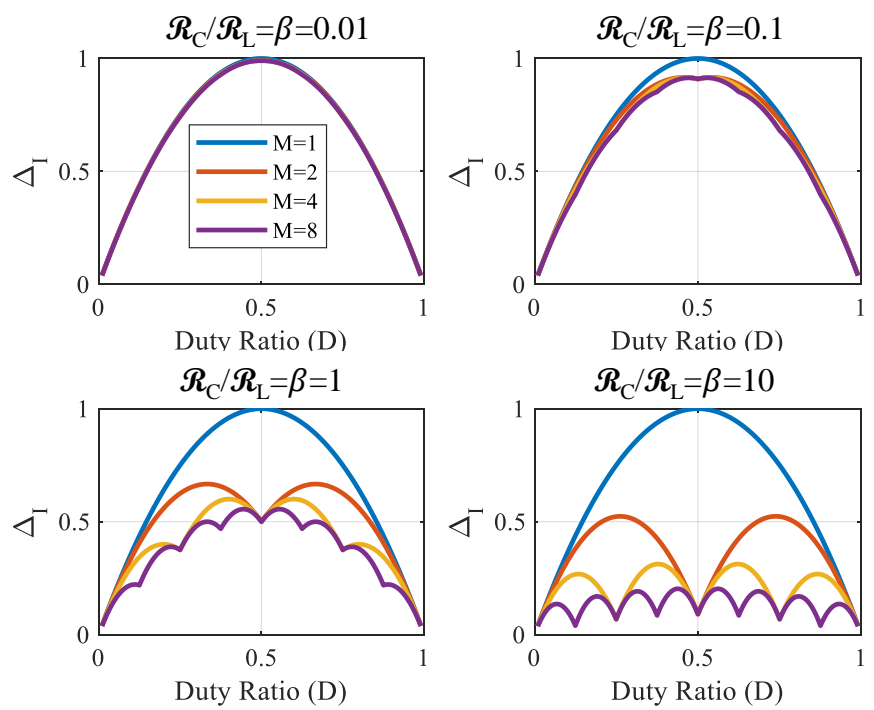

Fig. 26. Normalized winding current ripple $\left(\Delta_{I}\right)$ of the multiphase coupled buck inductor as functions of the number of phases $(M)$, duty ratio $(D)$, and reluctance ratio $\left(\beta=\mathcal{R}_{C} / \mathcal{R}_{L}\right)$. A lower $\Delta_{I}$ indicates smaller absolute winding current ripple. A high $\beta$ indicates strong coupling, and a low $\beta$ indicates weak coupling.

response $\left(L_{o t r}=L_{l} / M\right)$ and the overall output voltage ripple.

3) Determine the required value of $\mathcal{R}_{L}+M \mathcal{R}_{C}$ based on the selected $L_{p t r}=L_{l}$.

4) Design the magnetic structure (material and geometry) to minimize $\mathcal{R}_{L}$ and adjust $\mathcal{R}_{C}$ to maintain the selected $L_{p t r}$, optimize the loss, and ensure enough margin to avoid saturation under balanced excitation.

5) Evaluate the flux under the expected worst-case mismatch between phase currents. If this leads to saturation, add small gaps in the outer legs as necessary to accommodate the mismatch.

There is always parasitic inductance adding to $L_{l}$ of the coupled inductor. When the targeted value of $L_{p t r}=L_{l}$ is small, the parasitic inductance outside the transformer may provide a significant fraction of the necessary leakage inductance, thus providing an opportunity to reduce the required inductance and thus reduce the energy storage required in the magnetic structure and reduce its size. In some cases, the parasitic inductance may exceed the targeted value of $L_{l}$, and careful layout to reduce parasitic inductance may be needed.

The absolute value of the current ripple per-phase impacts the loss in the windings and switches.The maximum current ripple is useful for evaluating the performance of the system if $D$ spans across a wide range. When $D=0.5, M=1$, $k=0$, the effective inductance is $L_{p s s}=\frac{N^{2}}{\mathcal{R}_{C}+\mathcal{R}_{L}}$, leading to the maximum inductor current ripple per-phase:

$$
\Delta i_{\text {max }}=\frac{V_{I N} T\left(\mathcal{R}_{C}+\mathcal{R}_{L}\right)}{4 N^{2}} .
$$

The per-phase current ripple $\Delta i_{\text {phase }}$ is:

$\frac{V_{I N} D T}{N^{2}}\left(-\frac{k^{2} \mathcal{R}_{C}}{D M}-\frac{k \mathcal{R}_{C}}{D M}+2 k \mathcal{R}_{C}-D M \mathcal{R}_{C}+\mathcal{R}_{C}-D \mathcal{R}_{L}+\mathcal{R}_{L}\right)$.

The normalized per-phase current ripple $\left(\Delta_{I} \stackrel{\text { def }}{=} \frac{\Delta i_{\text {phase }}}{\Delta i_{\text {max }}}\right)$ is:

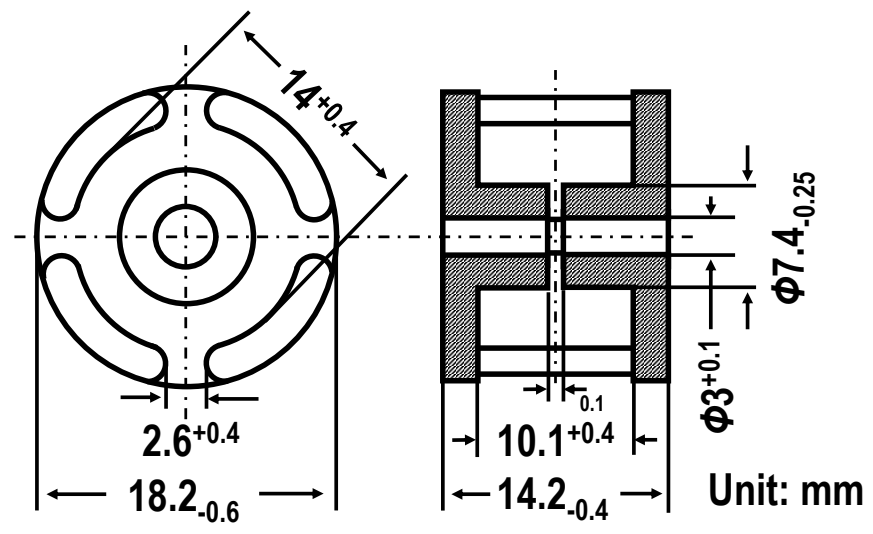

Fig. 27. The geometry of a four-phase coupled inductor with four side legs and one center leg (dimension labeled in $\mathrm{mm}$ ). The effective length of each side leg is $18 \mathrm{~mm}$. The effective area of each side leg is $11.25 \mathrm{~mm}^{2}$. The effective area of the center leg is $45 \mathrm{~mm}^{2}$. The relative permeability of the core is 2300 . There is a $0.1 \mathrm{~mm}$ air gap in the center leg.

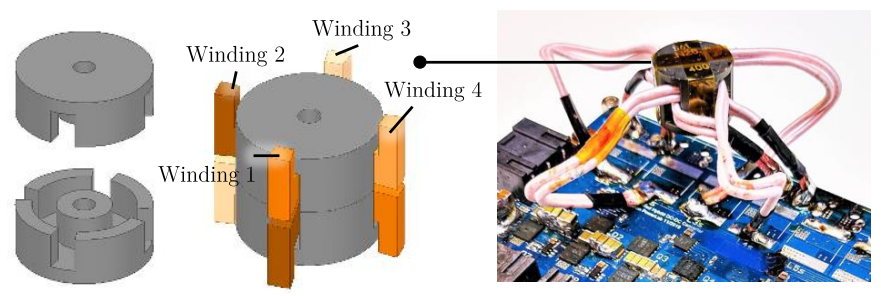

Fig. 28. An example four-phase coupled inductor buck converter. Each phase has a single turn. The extra winding outside the core (for current measurement) adds about $30 \mathrm{nH}$ parasitic inductance to each winding of the coupled inductor.

$$
\Delta_{I}=\frac{4 D\left(-\frac{k^{2} \beta}{D M}-\frac{k \beta}{D M}+2 k \beta-D M \beta+\beta-D+1\right)}{1+\beta} .
$$

Fig. 26 plots the normalized current ripple across a range of $D, M$, and $\beta$. Both Figs. 26 and 25 illustrate the advantages of increasing the coupling and of increasing the number of phases. Fig. 25 directly shows how much benefit is provided at a given operating point, whereas Fig. 26 is useful for considering a range of operating points with different duty ratios, and assessing the ripple and ripple reduction at the worst-case point over that range.

\section{MOdEL VerificATION}

A four-phase coupled inductor design was selected to verify the models discussed in this paper. This design is not optimized for a particular application. Fig. 27 shows the geometry of a SIEMENS P1814 B65561-A0400 core with N26 material. The structure has four side legs and one center leg.

The reluctances $\mathcal{R}_{L}$ and $\mathcal{R}_{L}$ were calculated based on simple approximations. These values, denoted as $\mathcal{R}_{L}^{*}$ and $\mathcal{R}_{C}^{*}$, are $492,070 \mathrm{H}^{-1}$ and $1,860,700 \mathrm{H}^{-1}$, respectively. Fig. 29 shows the measured inductance of the prototype following the parameter extraction method described in Appendix II using a HP/Agilent 4395A impedance analyzer. With $N=1$, the measured inductance with four windings connected in parallel $L_{o t r}$ was $25.7 \mathrm{nH}$. The measured self inductance $L_{S}$ was $1.54 \mu \mathrm{H}$. The extracted $\mathcal{R}_{L}$ was $496,100 \mathrm{H}^{-1}$, and $\mathcal{R}_{C}$ was 

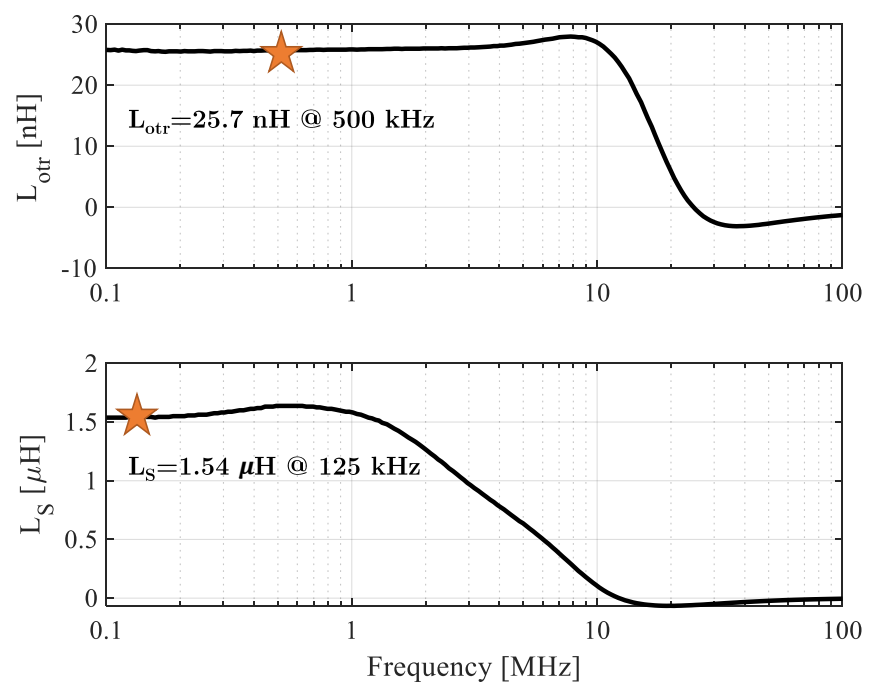

Fig. 29. Measurements used for parameter extraction from the fabricated coupled inductor. $L_{o t r}$ : measured inductance of four windings connected in parallel. $L_{S}$ : measured inductance of a single winding on the side leg.

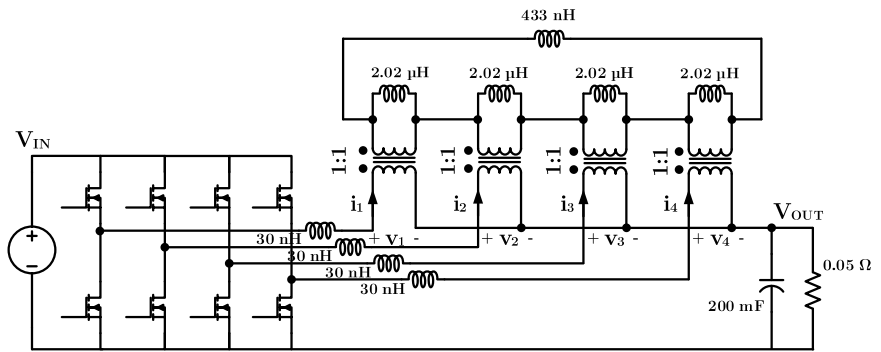

Fig. 30. SPICE simulation of the four-phase coupled inductor buck converter based on the inductance dual model.

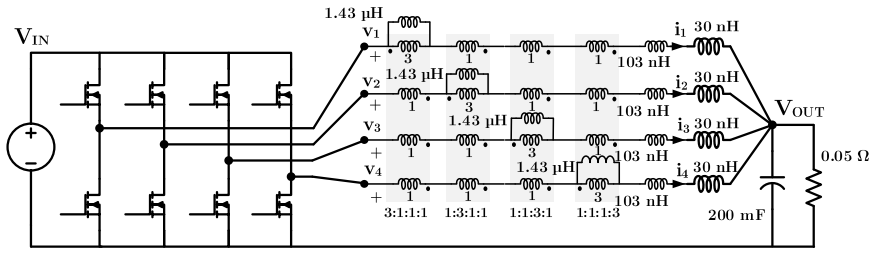

Fig. 31. SPICE simulation of the four-phase coupled inductor buck converter based on the multiwinding transformer model implemented with an ideal voltage equalization transformer.

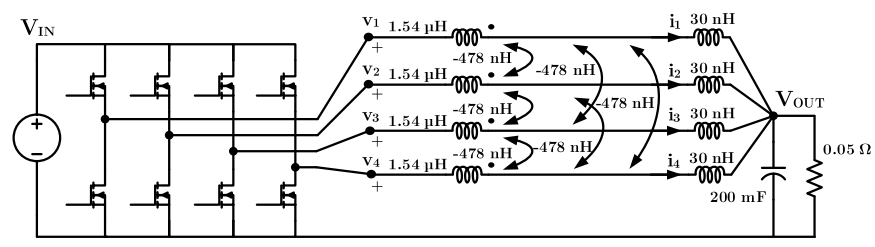

Fig. 32. SPICE simulation of the four-phase coupled inductor buck converter based on the inductance matrix model.

2,307,600 $\mathrm{H}^{-1}$. The extracted parameters match well with the calculated ones. We attribute the discrepancy in $\mathcal{R}_{C}$ to limited precision in fabricating the gap.

Fig. 33 shows the simulated open-loop startup voltage transient waveforms of three experimental designs. Design-1
TABLE II

Design Parameters of THE Prototype Coupled Inductor

\begin{tabular}{c||cc|cc}
\hline Measured & $L_{S}$ & $1.54 \mu \mathrm{H}$ & $L_{\text {otr }}$ & $25.7 \mathrm{nH}$ \\
Extracted & $\mathcal{R}_{L}$ & $496,100 \mathrm{H}^{-1}$ & $\mathcal{R}_{C}$ & $2,307,600 \mathrm{H}^{-1}$ \\
Calculated & $\mathcal{R}_{L}^{*}$ & $492,070 \mathrm{H}^{-1}$ & $\mathcal{R}_{C}^{*}$ & $1,860,700 \mathrm{H}^{-1}$ \\
\hline Inductance Dual & $L_{L}$ & $2.02 \mu \mathrm{H}$ & $L_{C}$ & $433 \mathrm{nH}$ \\
Multiwind. Xformer & $L_{l}$ & $103 \mathrm{nH}$ & $L_{\mu}$ & $1.43 \mu \mathrm{H}$ \\
Inductance Matrix & $L_{S}$ & $1.54 \mu \mathrm{H}$ & $L_{M}$ & $-478 \mathrm{nH}$ \\
\hline Parasitic Inductance & $\| 5$ \\
\hline Steady-state & $L_{o s s}$ & $332 \mathrm{nH}$ & $L_{p s s}$ & $838 \mathrm{nH}$ \\
Transient & $L_{\text {otr }}$ & $33.2 \mathrm{nH}$ & $L_{p t r}$ & $133 \mathrm{nH}$ \\
\hline FOM & $\|$ & Ripple Ratio: $15.9 \%$; Inductance Reduction: 6.3x \\
\hline
\end{tabular}

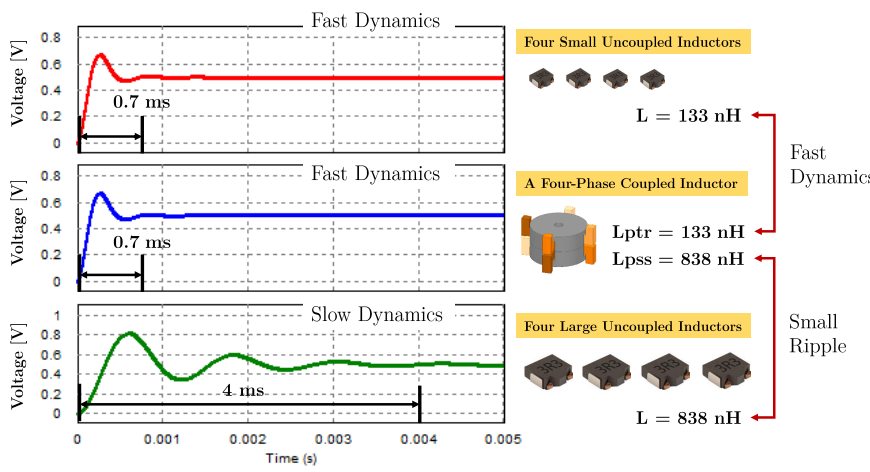

Fig. 33. Simulated transient output voltage waveforms of a four-phase buck converter with a) four uncoupled $133 \mathrm{nH}$ inductors; b) one four-phase coupled inductor with a per-phase transient inductance of $133 \mathrm{nH}$, and per-phase steady-state inductance of $838 \mathrm{nH}$; and c) four uncoupled $838 \mathrm{nH}$ inductors.

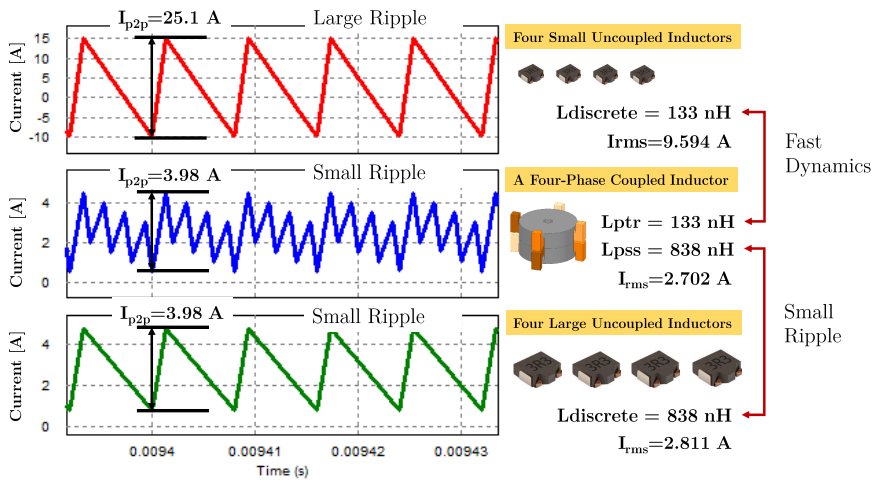

Fig. 34. Simulated steady-state winding current of a four-phase buck converter with a) four uncoupled $133 \mathrm{nH}$ inductors; b) one four-phase coupled inductor with a per-phase transient inductance of $133 \mathrm{nH}$, and per-phase steady-state inductance of $838 \mathrm{nH}$; and c) four uncoupled $838 \mathrm{nH}$ inductors.

comprises four small $133 \mathrm{nH}$ uncoupled inductors; Design2 comprises one four-phase coupled inductor with $133 \mathrm{nH}$ per-phase transient inductance and with coupling reducing the ripple to the equivalent of that expected with $838 \mathrm{nH}$ perphase inductance; and Design-3 comprises four big uncoupled $838 \mathrm{nH}$ inductors. The output voltage transient waveforms of Design-1 and Design-2 are exactly the same. The output voltage transient of Design-3 has large overshoot and oscillation.

The inductance dual model, multiwinding transformer model, and inductance matrix model of the prototype coupled inductor are shown in Figs. 30-32, respectively. In a 


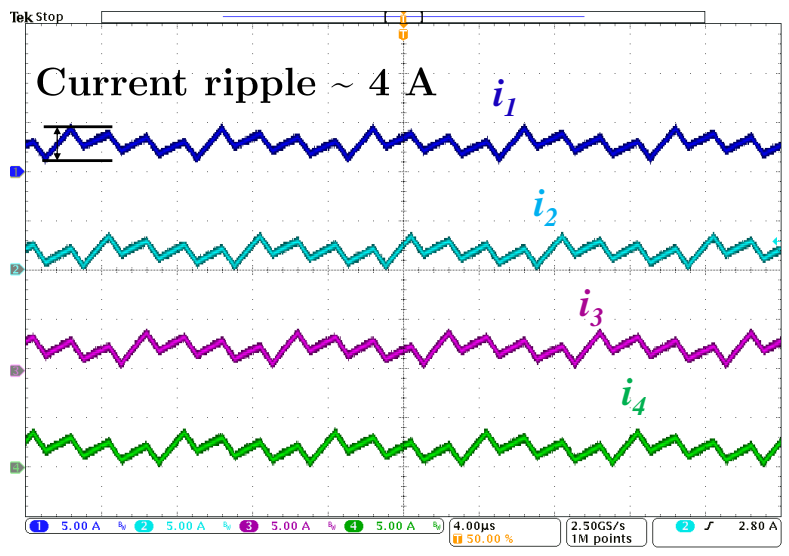

Fig. 35. Measured waveforms of the symmetric four-phase coupled inductor.

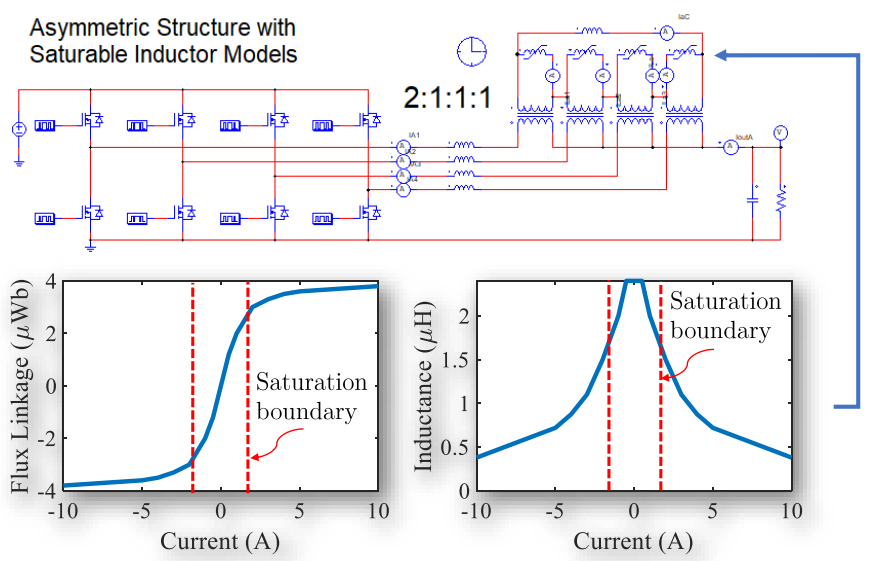

Fig. 36. Simulation setup in Powersim with asymmetric structure and saturable inductor models. The saturable inductor model represents non-linear current-flux-linkage relationships in circuit simulations.

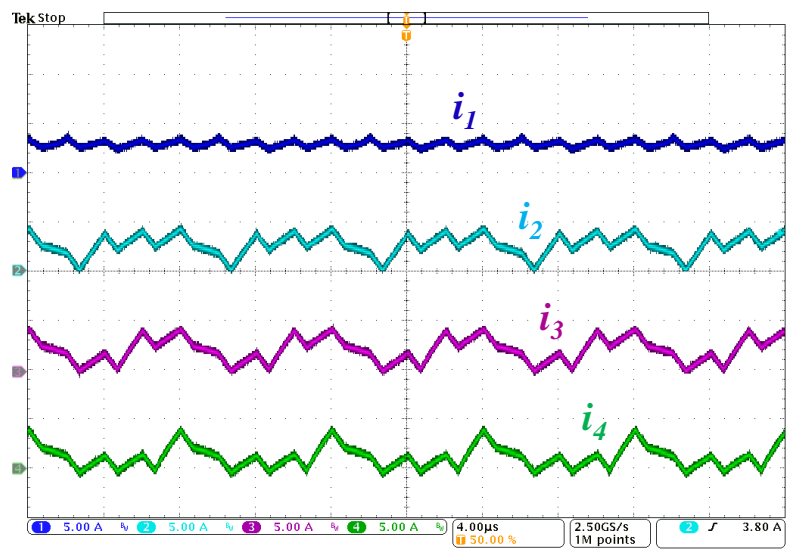

Fig. 37. Measured waveforms of the asymmetric four-phase coupled inductor. Winding \#1 has two turns, and Windings \#2-\#4 have a single turn.

multiwinding transformer model, $L_{l}$ is $133 \mathrm{nH}$ and $L_{\mu}$ is $1.43 \mu \mathrm{H}$. The input voltage is $3 \mathrm{~V}$, the average output current is $10 \mathrm{~A}$, the output capacitance is $200 \mathrm{mF}$, the output resistance is $0.05 \Omega$, the switching frequency is $125 \mathrm{kHz}$, and the duty ratio $D=1 / 6$. The extracted $L_{l}$ and $L_{\mu}$ were used to calculated $L_{o s s}, L_{p s s}, L_{o t r}, L_{p t r}$ as listed in Table II.

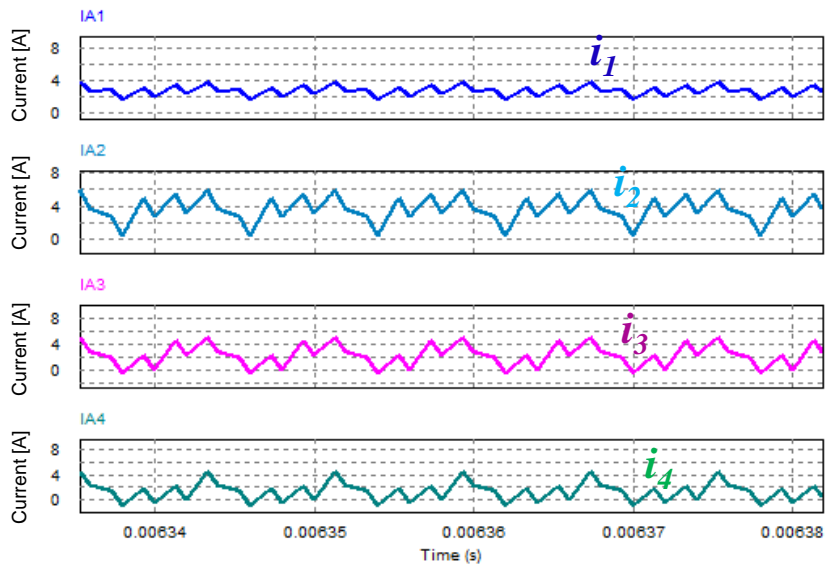

Fig. 38. Simulated waveforms of the asymmetric four-phase coupled inductor. Winding \#1 has two turns, and Windings \#2-\#4 have a single turn.

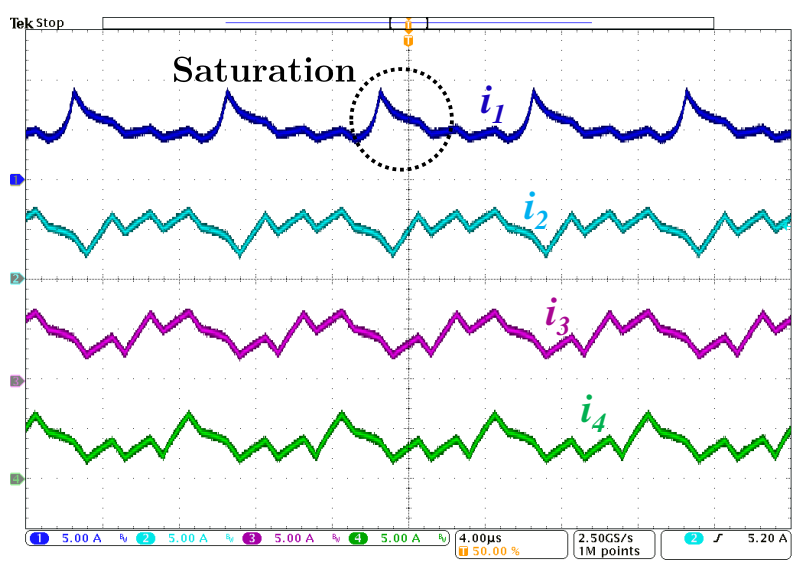

Fig. 39. Measured waveforms of the asymmetric coupled inductor with 2:1:1:1 turns ratio. The leg with two turns is saturated and a current spike is observed. The waveform patterns of the other three legs remain the same.

For an un-coupled four-phase buck converter with $133 \mathrm{nH}$ inductance per-phase, the current ripple per-phase is expected to be $25.1 \mathrm{~A}$. The expected rms current is $9.59 \mathrm{~A}$. With the prototype four-phase coupled inductor, the transient inductance per-phase is maintained at $133 \mathrm{nH}$, but the steady-state phase current ripple is reduced by coupling, to an expected value of $3.98 \mathrm{~A}, 15.8 \%$ of that of the uncoupled case. The expected rms current is $2.70 \mathrm{~A}, 28.1 \%$ of that of the uncoupled case. Fig. 34 shows the simulated steady-state per-phase current of the three converters. The per-phase current ripple of the converter with four small inductors was $25.1 \mathrm{~A}$, and the perphase current ripple of the buck converter with the coupled inductor was $3.98 \mathrm{~A}$. The simulation results match with the calculated results. Fig. 35 shows the measured waveforms of the experimental prototype. As expected, the measured perphase peak-to-peak current ripple is about $4 \mathrm{~A}$.

To demonstrate the capability of the inductance dual model to include magnetic saturation, the design was modified by changing the number of turns on one of the windings to two, while leaving the others windings with one turn. This results in an asymmetric structure that saturates early with equal dc currents in the different legs. Fig. 36 shows a circuit 


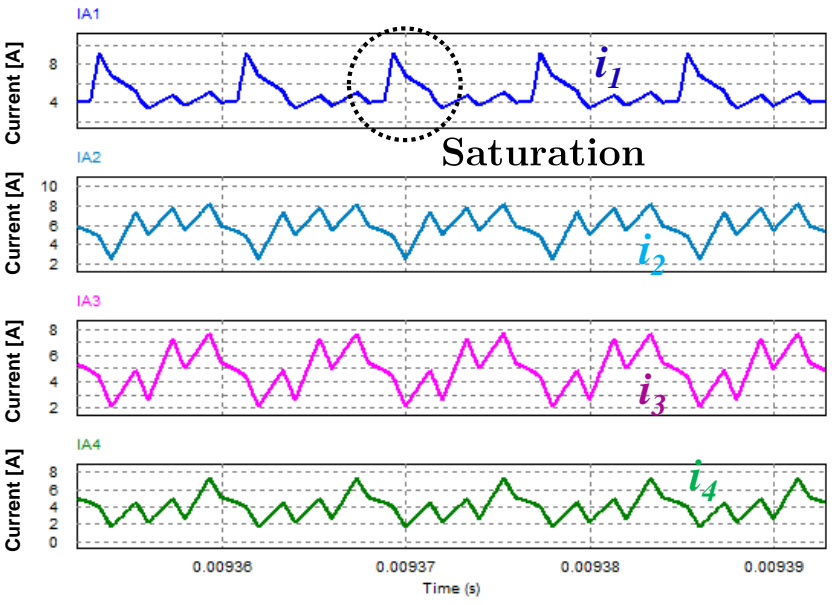

Fig. 40. Simulated waveforms of the asymmetric coupled inductor with 2:1:1:1 turns ratio.The leg with two turns is saturated and a current spike is observed. The waveform patterns of the other three legs remain the same.

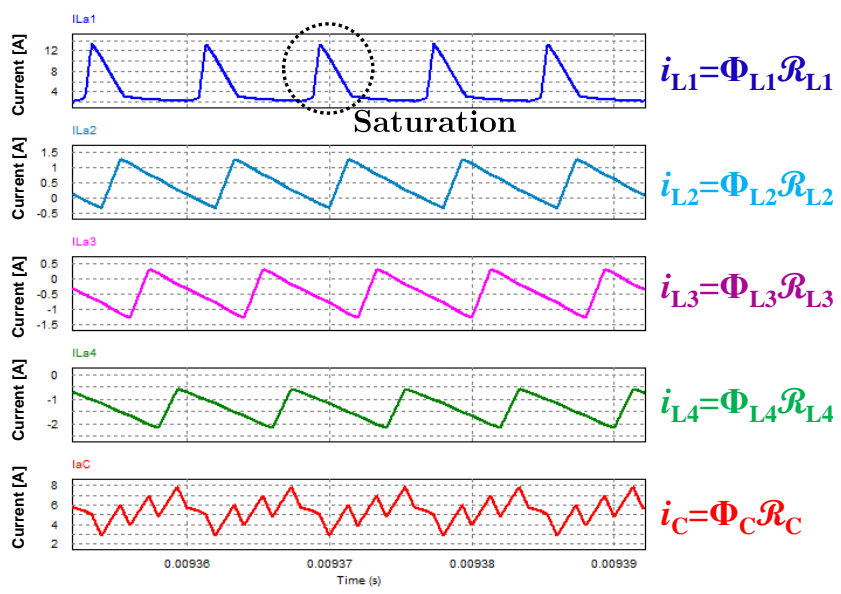

Fig. 41. Simulated current waveforms of $L_{L}$ and $L_{C}$ in the inductance dual model when the inductors $L_{L}$ are implemented as saturable inductors. The leg with two turns is saturated. The inductance dual model allows the magnetic flux be visualized as current, and enable rapid evaluation of the core flux density.

simulation model (implemented in Powersim) with saturable inductor models. A non-linear current-flux-linkage relationship was implemented in the saturable inductor model in circuit simulations. The unbalanced MMF leads to unbalanced current and flux distribution, and may cause saturation when the flux mismatch is significant. Fig. 37 shows the measured waveforms of the modified design with $10 \mathrm{~A}$ as the overall output current. Strong asymmetry was observed in phase current, as predicted by the simulation results in Fig. 38.

The saturation flux density $B_{\text {sat }}$ of the SIEMENS N26 material is about $390 \mathrm{mT}$ at $25^{\circ} \mathrm{C}$. The saturation flux $\Phi_{\text {sat }}=$ $B_{\text {sat }} A_{C}$ of the side leg was $4.388 \mu \mathrm{Wb}\left(A_{C}=11.25 \mathrm{~mm}^{2}\right)$. $\mathcal{R}_{L}$ was $496,100 \mathrm{H}^{-1}$. The threshold current for the saturable inductor is about 2.17 A. Fig. 39 shows the measured current waveforms when winding \#1 with two turns is saturated $\left(i_{1}\right)$. The total output current is about $20 \mathrm{~A}$. The average current per phase is about $5 \mathrm{~A}$. The per-phase inductance of this winding drops significantly as the inductor current $\left(i_{1}\right)$ approaches the saturation limit $(5 \mathrm{~A})$, which matches well with

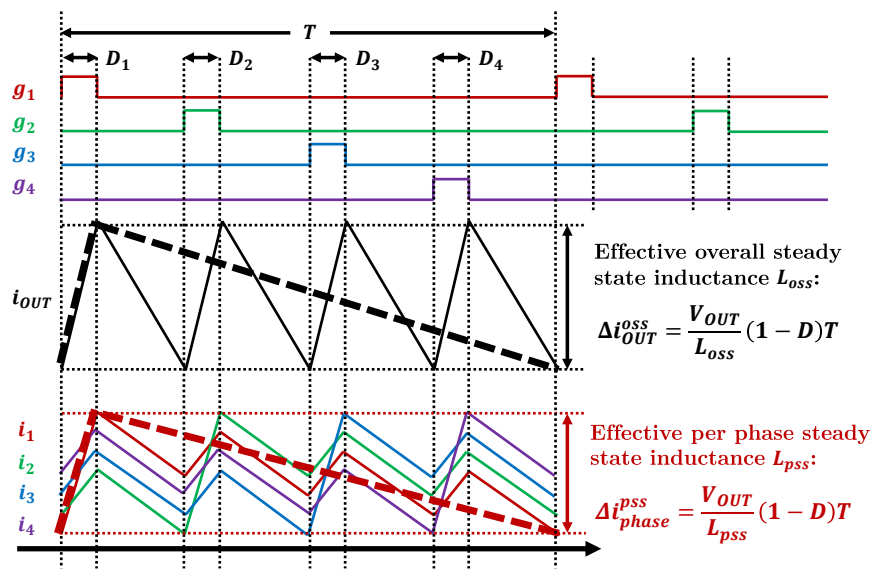

Fig. 42. Effective overall steady-state inductance $\left(L_{o s s}\right)$ and effective perphase steady-state inductance $\left(L_{p s s}\right)$ of the multiphase coupled inductor.

the simulated results in Fig. 40. Fig. 41 shows the simulated current of $L_{L}$ and $L_{C}$ which reflects the side and center leg flux $\left(i_{L}=\Phi_{L} \mathcal{R}_{L}\right)$. The magnetic core was saturated when $i_{L}$ approaches $2.17 \mathrm{~A}\left(\Phi_{\text {sat }} \mathcal{R}_{L}\right)$. The non-linear saturation effects are captured in SPICE simulations.

\section{CONCLUSIONS}

This paper unifies the lumped circuit models for multiphase coupled inductors. Models including inductance matrix models, multiwinding transformer models, extended cantilever models, magnetic circuit models, gyrator-capacitor models, and inductance dual models are summarized and compared. These models represent identical mathematical relationships in the multiphase coupled inductors, but reveal different physical fundamentals and distinct design insights. Inductance matrix models, multiwinding transformer models, and extended cantilever models are math-based models. Magnetic circuit models, gyrator-capacitor models, and inductance dual models are physics-based models. The inductance dual model is particularly useful amongst these models because it is directly built on elements in the magnetic circuit model and offers convenience in circuit simulations. Core loss and saturation effects in each portion of the core can be captured. These models and design equations are verified through theoretical derivation, SPICE simulations, and experimental measurements.

\section{ACKNOWLEDGEMEnts}

This work was supported by the the National Science Foundation under Award \#1847365. The authors would like to thank Dr. Yenan Chen of Princeton University for collecting the experimental data of this work during COVID-19.

\section{APPendix I: Performance Parameters}

Several performance parameters are derived here using the inductance dual model. The results are equivalent to the expressions for the same parameters in [6], derived based on the inductance matrix model. 


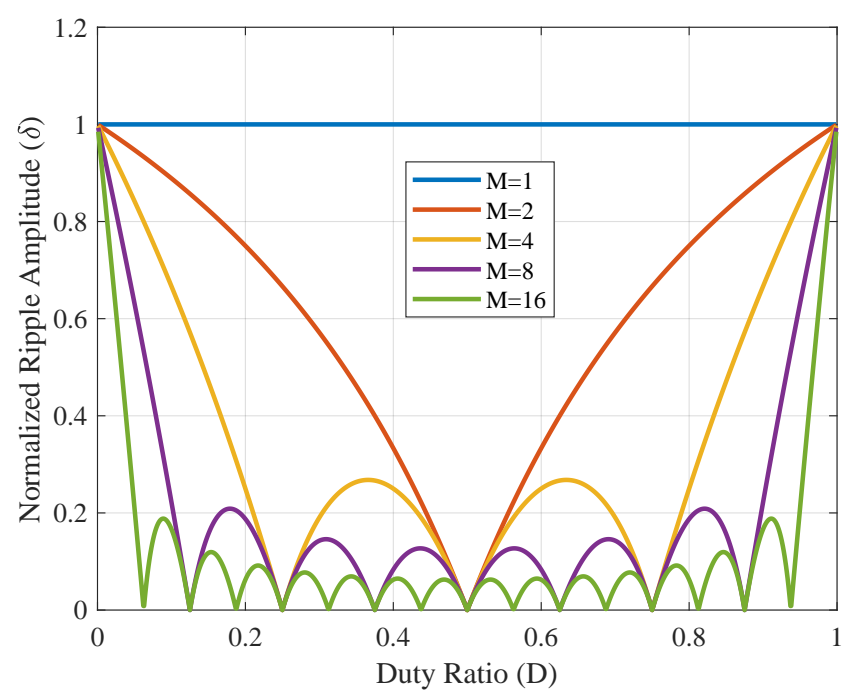

Fig. 43. Normalized ripple current amplitude $(\delta)$ of a $M$-phase interleaved uncoupled buck converter with $\frac{k}{M}<D<\frac{k+1}{M}$. The interleaving current ripple reduction mechanism contributes to reducing the overall output current ripple of the multiphase coupled inductor.

\section{A. Effective Overall Steady-State Inductance ( $\left.L_{\text {oss }}\right)$}

The effective overall steady-state inductance can be used to predict the effective output current ripple of the multiphase buck converter as if the multiphase coupled inductor is one single discrete inductor (Fig. 42). In a $M$ phase coupled inductor buck converter with $V_{I N}$ as the input voltage, an arbitrary duty ratio $\frac{k}{M}<D<\frac{k+1}{M}$, and $V_{O U T}=D V_{I N}$ as the output voltage, the derivative of the overall output current is the summation of the derivative of the current of all phases:

$$
\frac{d i_{O U T}}{d t}=\frac{d i_{1}}{d t}+\frac{d i_{2}}{d t}+\ldots+\frac{d i_{M}}{d t} .
$$

- During $\frac{k}{M} T<t<D T$, the overall current ramps up. There are always $k+1$ inductors which have $V_{I N}-V_{O U T}$ across them, and $M-k-1$ inductors which have $-V_{O U T}$ across them for an on period $\left(D-\frac{k}{M}\right) T$. The $\{k+1\}^{t h}$ inductor has $V_{I N}-V_{O U T}$ across it.

- During $D T<t<\frac{k+1}{M} T$, the overall current ramps down. There are always $i$ inductors which have $V_{I N}-V_{O U T}$ across them, and $M-k$ inductors which have $-V_{O U T}$ across them for an off period $\left(\frac{k+1}{M}-D\right) T$. The $\{k+1\}^{t h}$ inductor has $-V_{O U T}$ across it.

Substituting Eq. (30) into Eq. (6), we find that the current ripple during the ramp up and ramp down period are:

$$
\begin{aligned}
\Delta i_{\text {OUT }}^{u p} & =\frac{\left(\mathcal{R}_{L}+M \mathcal{R}_{C}\right)(k+1-D M)(D M-k)}{D M N^{2}} V_{O U T} T \\
\Delta i_{\text {OUT }}^{\text {down }} & =-\frac{\left(\mathcal{R}_{L}+M \mathcal{R}_{C}\right)(k+1-D M)(D M-k)}{D M N^{2}} V_{O U T} T .
\end{aligned}
$$

Note that in steady-state, $\Delta i_{\text {OUT }}^{s s}=\Delta i_{\text {OUT }}^{u p}=-\Delta i_{\text {OUT }}^{\text {down }}=$ $\frac{V_{O U T}}{L_{s s}}(1-D) T$. A single phase inductor with the same ripple would have a value

$$
L_{\text {oss }}=\underbrace{\frac{N^{2}}{\mathcal{R}_{L}+M \mathcal{R}_{C}}}_{L_{p t r}} \times \underbrace{\frac{(1-D) D M}{(k+1-D M)(D M-k)}}_{1 / \delta} .
$$

for an $M$ phase coupled buck converter with $\frac{k}{M}<D<\frac{k+1}{M}$. Here $L_{p t r}=\frac{N^{2}}{\mathcal{R}_{L}+M \mathcal{R}_{C}}$ is the equivalent inductance of the side leg of the coupled inductor as if the center leg is evenly divided into $M$ pieces. It is also the per-phase transient inductance as will be derived. Appendix II introduced a method to measure $L_{p t r} . \delta=\frac{(k+1-D M)(D M-k)}{(1-D) D M}$ is the normalized ripple amplitude of an uncoupled interleaved $M$-phase buck converter with duty ratio $D$ (Fig. 43) [50]-[52].

For an $M$-phase coupled inductor buck converter operating in continous conduction mode with duty ratio $D$, switching period $T$, and output voltage $V_{O U T}$, the peak-to-peak ripple of the overall output current is a simple function of $L_{o s s}$ :

$$
\Delta i_{O U T}=\frac{V_{O U T}(1-D) T}{L_{o s s}} .
$$

\section{B. Effective Per-Phase Steady-State Inductance $\left(L_{p s s}\right)$}

If $\frac{k}{M}<D<\frac{k+1}{M}$, during the $0<t<D T$ period, for each phase, there are $k+1$ numbers of $\left(D-\frac{k}{M}\right) T$ sub-periods, in which $k+1$ phases have $V_{I N}-V_{O U T}$ across them, and $M-k-1$ phases have $-V_{O U T}$ across them. The per-phase current ramps up. The current ripple in each winding during the $k+1$ numbers of $\left(D-\frac{k}{M}\right) T$ sub-periods is:

$$
\begin{aligned}
\Delta i_{\text {phase }}^{u p}= & \left(\left(\mathcal{R}_{L}+\mathcal{R}_{C}+k \mathcal{R}_{C}\right) \frac{1-D}{D}-(M-k-1) \mathcal{R}_{C}\right) \\
& \times\left(D-\frac{k}{M}\right) \frac{(k+1) T V_{\text {OUT }}}{N^{2}} .
\end{aligned}
$$

During the $k$ numbers of $\left(\frac{k+1}{M}-D\right) T$ subperiods, $k$ phases have $V_{I N}-V_{O U T}$ across them, and $M-k$ phases have $-V_{O U T}$ across them. The per-phase current ramps down. The current ripple in each phase during the $k$ numbers of $\left(\frac{k+1}{M}-D\right) T$ subperiod is:

$$
\begin{aligned}
\Delta i_{\text {phase }}^{\text {down }}= & \left(\left(\mathcal{R}_{L}+\mathcal{R}_{C}+(k-1) \mathcal{R}_{C}\right) \frac{1-D}{D}-(M-k) \mathcal{R}_{C}\right) \\
& \times\left(\frac{k+1}{M}-D\right) \frac{k T V_{\text {OUT }}}{N^{2}} .
\end{aligned}
$$

The current ripple in each phase is the summation of all the ramp up and ramp down sub-periods:

$\Delta i_{\text {phase }}^{\text {ss }}=\Delta i_{\text {phase }}^{\text {up }}+\Delta i_{\text {phase }}^{\text {down }}=$
$\frac{T V_{\text {OUT }}}{N^{2}}\left(-\frac{k^{2} \mathcal{R}_{C}}{D M}-\frac{k \mathcal{R}_{C}}{D M}-D \mathcal{R}_{L}-D M \mathcal{R}_{C}+2 k \mathcal{R}_{C}+\mathcal{R}_{L}+\mathcal{R}_{C}\right)$.

We define $L_{p s s}$ as the effective inductance of each winding in steady-state $\Delta i_{\text {phase }}^{s s}=\frac{V_{O U T}}{L_{p s s}}(1-D) T$ :

$$
L_{p s s}=\frac{N^{2}(1-D)}{-\frac{k^{2} \mathcal{R}_{C}}{D M}-\frac{k \mathcal{R}_{C}}{D M}+2 k \mathcal{R}_{C}-D M \mathcal{R}_{C}+\mathcal{R}_{C}-D \mathcal{R}_{L}+\mathcal{R}_{L}} .
$$

For an $M$-phase coupled inductor buck converter operating in continuous conduction mode with duty ratio $D$, switching period $T$, and output voltage $V_{O U T}$, the peak-to-peak current ripple of the per-phase winding current is:

$$
\Delta i_{\text {per-phase }}=\frac{V_{\text {OUT }}(1-D) T}{L_{p s s}} .
$$




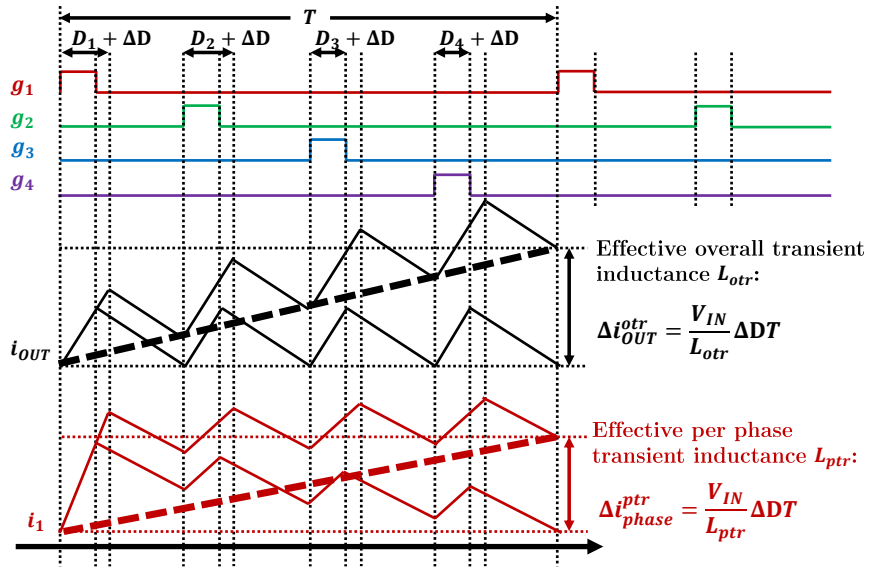

Fig. 44. Effective overall transient inductance $\left(L_{o t r}\right)$ and effective per-phase transient inductance $\left(L_{p t r}\right)$ of the multiphase coupled inductor.

\section{Effective Overall Transient Inductance ( $\left.L_{\text {otr }}\right)$}

The transient inductance determines the response of $i_{O U T}$ to a small perturbation in $D$. For a buck converter with a discrete inductor, the ramp up slope of the inductor current is $\frac{d i O U T}{d t}_{u p}=\frac{V_{I N}-V_{O U T}}{L}$, the ramp down slope of the inductor current is $\frac{d i_{O U T}}{d t}$ down $=\frac{-V_{O U T}}{L}$. If there is a small perturbation $\Delta D, i_{O U T}$ will ramp up $\frac{V_{I N}-V_{O U T}}{L} \Delta D T$ more, and will ramp down $\frac{V_{O U T}}{L} \Delta D T$ less. As a result, the perturbation in the inductor current is the summation of them, which is $\frac{V_{I N}}{L} \Delta D T$. In other words, the transient inductance of a singlephase uncoupled buck converter with $L$ is still $L$. For an $M$ phase coupled inductor buck converter, if $\frac{k}{M}<D<\frac{k+1}{M}$, the ramp up and ramp down rates are:

$$
\begin{aligned}
& \frac{d i_{\text {OUT }}^{t r-u p}}{d t}=\frac{\left(\mathcal{R}_{L}+M \mathcal{R}_{C}\right)(k+1-D M)}{D N^{2}} V_{O U T}, \\
& \frac{d i_{\text {OUT }}^{\text {tr-down }}}{d t}=\frac{\left(\mathcal{R}_{L}+M \mathcal{R}_{C}\right)(k-D M)}{D N^{2}} V_{O U T} .
\end{aligned}
$$

Note that there are $M$ ripple cycles within a switching period $T$. A perturbation $\Delta D$ results in a perturbation of $\left(\frac{d i_{O U T}^{u p}}{d t}-\frac{d i_{O U T}^{d o w n}}{d t}\right) M T \Delta D$, which equals $\frac{\left(\mathcal{R}_{L}+M \mathcal{R}_{C}\right)}{N^{2}} V_{I N} M T \Delta D$ in the overall output current. Since

$$
\Delta i_{\text {OUT }}^{t r}=\frac{V_{I N}}{L_{\text {otr }}} \Delta D T=\frac{\left(\mathcal{R}_{L}+M \mathcal{R}_{C}\right)}{N^{2}} V_{I N} M \Delta D T,
$$

the effective overall transient inductance of a $M$ phase coupled inductor, regardless of the duty ratio $D$, is:

$$
L_{\text {otr }}=\frac{N^{2}}{M\left(\mathcal{R}_{L}+M \mathcal{R}_{C}\right)} .
$$

$L_{\text {otr }}$ is effectively connecting $M$ discrete inductors, each of reluctance value $\mathcal{R}_{L}+M \mathcal{R}_{C}$ in parallel.

\section{Effective Per-Phase Transient Inductance $\left(L_{p t r}\right)$}

Since the current equally distribute among the $M$ parallel windings, with a purtubation of $\Delta D$ in the duty ratio, the perturbation of the current in each winding is $\frac{1}{M}$ of the overall current perturbation. As a result:

$$
\frac{V_{I N}}{L_{t r w}} \Delta D T=M \frac{\left(\mathcal{R}_{L}+M \mathcal{R}_{C}\right)}{N^{2}} V_{I N} \Delta D T \times \frac{1}{M} .
$$

The effective per-phase transient inductance of a $M$ phase coupled inductor, regardless of the duty ratio $D$, is:

$$
L_{p t r}=\frac{N^{2}}{\mathcal{R}_{L}+M \mathcal{R}_{C}} .
$$

\section{ApPendix II: Model Parameter Extraction}

This Appendix introduces a method to extract parameters for the inductance dual model from impedance measurements. By connecting an $\mathrm{N}$-turn winding to one of the side legs and measuring the inductance, we can measure the self inductance $\left(L_{S}\right)$ of one winding:

$$
L_{S}=L_{\mu}+L_{l}=N^{2} \frac{\mathcal{R}_{L}+(M-1) \mathcal{R}_{C}}{\mathcal{R}_{L}\left(\mathcal{R}_{L}+M \mathcal{R}_{C}\right)} .
$$

By connecting all windings in parallel, we can measure the overall transient inductance $\left(L_{o t r}\right)$ of the coupled inductor:

$$
L_{\text {otr }}=\frac{L_{l}}{M}=\frac{N^{2}}{M\left(\mathcal{R}_{L}+M \mathcal{R}_{C}\right)} .
$$

$\mathcal{R}_{L}$ and $\mathcal{R}_{C}$ can be found from the measured $L_{S}, L_{o t r}$, and the known values of $N$ and $M$ :

$$
\begin{gathered}
\mathcal{R}_{L}=\frac{N^{2}(M-1)}{M\left(L_{S}-L_{o t r}\right)}, \\
\mathcal{R}_{C}=\frac{N^{2}\left(L_{S}-M L_{o t r}\right)}{M^{2} L_{o t r}\left(L_{S}-L_{o t r}\right)} .
\end{gathered}
$$

\section{REFERENCES}

[1] D. C. Prince, "Rectifier voltage control," Journal of the A.I.E.E., vol. 45, no. 7, pp. 630-636, July 1926.

[2] S. Cuk, "New magnetic structures for switching converters," IEEE Trans. on Magnetics, vol. 19, no. 2, pp. 75-83, March 1983.

[3] A. Dauhajre, "Modelling and estimation of leakage phenomena in magnetic circuits," Ph.D. thesis, California Institute of Technology, Pasadena, CA, Apr. 1986.

[4] P.-L. Wong, "Performance improment of multi-channel interleaving voltage regulator modules with integrated coupling inductors", Ph.D. thesis, Virginia Tech, Blacksburg, VA, Mar., 2001.

[5] W. Chen, "Low voltage high current power conversion with integrated magnetic," Ph.D. thesis, Virginia Tech, Blacksburg, VA, Apr. 27, 1998.

[6] Y. Dong, "Investigation of multiphase coupled-inductor buck converters in point-of-load applications", PhD Thesis, Virginia Tech, 2009.

[7] J. G. Kassakian, M. F. Schlecht, and G. C. Verghese, "Magnetic Components," in Principles of Power Electronics, AddisonWesley, 1991.

[8] P. T. Krein, "Concepts of Magnetics for Power Electronics," in Elements of Power Electronics, Vol. 126. New York: Oxford University Press, 1998.

[9] R. W. Erickson and D. Maksimovic, "Basic Magnetics Theory," in Fundamentals of Power Electronics, Boston, MA, USA: Kluwer, 2001.

[10] R. W. Erickson and D. Maksimovic, "A multiple-winding magnetics model having directly measurable parameters," Proc. of the IEEE Power Electron. Special. Conf. (PESC), vol.2, pp.14721478, 17-22, May 1998.

[11] Q. Chen, F. C. Lee, J. Z. Jiang and M. M. Jovanovic, "A new model for multiple-winding transformer," Proceedings of 1994 Power Electronics Specialist Conference, Taipei, Taiwan, 1994, pp. 864-871 vol.2.

[12] MIT EE Staff, Magnetic Circuits and Transformers, Cambridge MA: The MIT Press, 1943. 
[13] H. A. Haus and J. R. Melcher, "Introduction to Electroquasistatics and Magnetoquasistatics", in Electromagnetic Fields and Energy, Englewood Cliffs, NJ: Prentice-Hall, 1989.

[14] J. M. Alonso, G. Martinez, M. Perdigao, M. R. Cosetin and R. N. do Prado, "A Systematic Approach to Modeling Complex Magnetic Devices Using SPICE: Application to Variable Inductors," IEEE Transactions on Power Electronics, vol. 31, no. 11, pp. 7735-7746, Nov. 2016.

[15] D. C. Hamill, "Lumped equivalent circuits of magnetic components: the gyrator-capacitor approach," IEEE Transactions on Power Electronics, vol. 8, no. 2, pp. 97-103, April 1993.

[16] D. C. Hamill, "Gyrator-capacitor modeling: a better way of understanding magnetic components," Proceedings of 1994 IEEE Applied Power Electronics Conference and Exposition, Orlando, FL, USA, 1994, pp. 326-332.

[17] S. El-Hamamsy and E. I. Chang, "Magnetics modeling for computer-aided design of power electronics circuits," IEEE Power Electronics Specialists Conference, Milwaukee, WI, USA, 1989, pp. 635-645 vol.2.

[18] G. W. Ludwig and S. El-Hamamsy, "Coupled inductance and reluctance models of magnetic components," IEEE Transactions on Power Electronics, vol. 6, no. 2, pp. 240-250, April 1991.

[19] I. G. Park and S. I. Kim, "Modeling and analysis of multiinterphase transformers for connecting power converters in parallel," IEEE Power Electronics Specialists Conference, Saint Louis, MO, USA, 1997, pp. 1164-1170, vol.2.

[20] B. Cougo, "Design and Optimization of Inter Cell Transformers for Parallel MultiCell Converters," Ph.D. Thesis, University de Toulouse, 2011.

[21] F. M. Lambert, J. Mahseredjian, M. Martinez-Duro and F. Sirois, "Magnetic circuits within electric circuits: critical review of existing methods and new mutator implementations," IEEE Transactions on Power Delivery, vol. 30, no. 6, pp. 2427-2434, Dec. 2015.

[22] S. M. Sandler, "SPICE Modeling of Magnetic Components," Switch-Mode Power Supply Simulation: Designing with SPICE 3, McGraw-Hill, 2006.

[23] M. Chen, M. Araghchini, K. K. Afridi, J. H. Lang, C. R. Sullivan and D. J. Perreault, "A systematic approach to modeling impedances and current distribution in planar magnetics," IEEE Trans. on Power Electron., vol. 31, no. 1, pp. 560-580, Jan. 2016.

[24] A. M. Urling, V. A. Niemela, G. R. Skutt, and T. G. Wilson, "Characterizing high-frequency effects in transformer windings-A guide to several significant articles," Proc. IEEE Appl. Power Electron. Conf. Expo., Mar. 13-17, 1989, pp. 373-385.

[25] V. A. Niemela, G. R. Skutt, A. M. Urling, Y.-N. Chang, T. G. Wilson, Jr., H. A. Owen, and R. C. Wong, "Calculating the short-circuit impedances of a multiwinding transformer from its geometry," IEEE Power Electron. Spec. Conf., Jun. 26-29, 1989, vol. 2, pp. 607-617.

[26] C. Zhao, S. D. Round, and J. W. Kolar, "An Isolated Three-Port Bidi-rectional DC-DC Converter with Decoupled Power Flow Management," IEEE Trans. Power Electron., vol. 23, no. 5, pp. 2443-2453, Sep. 2008.

[27] Y. Chen, P. Wang, Y. Elasser and M. Chen, "Multicell Reconfigurable Multi-Input Multi-Output Energy Router Architecture," IEEE Transactions on Power Electronics, vol. 35, no. 12, pp. 13210-13224, Dec. 2020.

[28] P. Wang, Y. Chen, J. Yuan, R. C. N. Pilawa-Podgurski, M. Chen, "Differential Power Processing for Ultra-Efficient Data Storage," IEEE Transactions on Power Electronics, accepted.

[29] F. E. Terman, "Section 2: Circuit Elements," in Radio Engineers' Handbook, First Edition, New York, NY: McGraw-Hill Book Company, 1943.

[30] W. H. Hayt and J. E. Kemmerly, "Inductance and Capacitance: Duality," in Engineering Circuit Analysis, Fourth Edition, New York: McGraw-Hill Book Company, 1993.

[31] E. C. Cherry, "The duality between interlinked electric and magnetic circuits and the formation of transformer equivalent circuits," Proceedings of the Physical Society, vol. 62 part 2, section B, no. 350 B, Feb. 1949, pp. 101-111.

[32] L. O. Chua, Linear and Non-linear Circuits. New York: McGraw-Hill, 1987, pp. 469-472.

[33] A. V. Ledenev, G. G. Gurov, and R. M. Porter, "Multiple Power Converter System Using Combining Transformers", US Patent US 6,545,450 B1, April 8, 2003.

[34] P. Zumel, O. Garcia, J. Cobos, J. Uceda,"Magnetic integration for interleaved converters", IEEE Applied Power Electronics Conference, 2003.

[35] P. Zumel, O. Garcia, J. A. Cobos and J. Uceda, “Tight magnetic coupling in multiphase interleaved converters based on simple transformers," IEEE Applied Power Electron. Conf., 2005.

[36] Z. Lu, W. Chen, "Multi-phase inductor coupling scheme with balancing winding in VRM applications", in IEEE Applied Power Electronics Conference, 2007, pp.731-735.

[37] H. A. Owen, V. A. Niemela and T. G. Wilson, "Enhanced crosscoupled-secondaries model for multiwinding transformers," IEEE Power Electronics Specialists Conference, Toledo, Spain, 1992, pp. 1269-1276 vol.2, doi: 10.1109/PESC.1992.254729.

[38] J. G. Hayes, N. O'Donovan and M. G. Egan, "The extended T model of the multiwinding transformer," IEEE Power Electronics Specialists Conference, 2004, pp. 1812-1817.

[39] B. Hesterman, "Analysis and modeling of magnetic coupling", Denver Chapter IEEE Power Electron. Soc., 2007.

[40] A. M. Schultz and C. R. Sullivan, "Voltage converter with coupled inductive windings, and associated methods," U.S. Patent 6,362,986, March 26, 2002.

[41] J. Li, T. Abdallah, and C. R. Sullivan, "Improved calculation of core loss with non-sinusoidal waveforms", IEEE IAS, 2001.

[42] J. Li, C. R. Sullivan, and A. Schultz, "Coupled inductor design optimization for fast-response low-voltage DC-DC converters," IEEE Applied Power Electronics Conference and Exposition, APEC 2002, pp. 817-823 vol.2.

[43] J. Li, A. Stratakos, C. R. Sullivan, and A. Schultz, "Using coupled inductors to enhance transient performance of multi-phase buck converters," IEEE Applied Power Electronics Conference and Exposition, APEC 2004, pp. 1289-1293 vol.2.

[44] T. Schmid, A. Ikriannikov, "Magnetically coupled buck converters," IEEE Energy Conversion Congress and Exposition, Denver CO, pp. 4948-4954, 2013.

[45] A. Ikriannikov, "The Benefits of the Coupled Inductor Technology," Tutorial 5997, Maxim Integrated, 2014.

[46] X. Zhou, P.-L. Wong, P. Xu, F. C. Lee, and A. Q. Huang, "Investigation of candidate VRM topologies for future microproceossrs," IEEE Trans. Power Electron., vol. 15, Nov. 2000.

[47] P.-L. Wong, P. Xu, P. Yang, and F. C. Lee, "Performance improvements of interleaving VRMs with coupling inductors," IEEE Trans. on Power Electron., vol. 16, no. 4, 2001.

[48] P.-L. Wong, F. C. Lee, P. Xu and K. Yao, "Critical inductance in voltage regulator modules," IEEE Transactions on Power Electronics, vol. 17, no. 4, pp. 485-492, July 2002.

[49] P. Xu, J. Wei, K. Yao, Y. Meng, F. C. Lee, "Investigation of candidate topologies for 12 V VRM," IEEE Applied Power Electronics Conference and Exposition, pp. 686-692, 2002.

[50] B. Miwa,"Interleaved Conversion Techniques for High Density Power Supplies," PhD Thesis, Massachusetts Institute of Technology, 1992.

[51] C. Chang and M. A. Knights, "Interleaving technique in distributed power conversion systems," IEEE Transactions on Circuits and Systems I: Fundamental Theory and Applications, vol. 42, no. 5, pp. 245-251, May 1995.

[52] D. J. Perreault and J. G. Kassakian, "Distributed interleaving of paralleled power converters," IEEE Transactions on Circuits and Systems I: Fundamental Theory and Applications, vol. 44, no. 8, pp. 728-734, Aug. 1997.

[53] S. R. Lee, H. Li, D. Zhou, and M. Chen, "Princeton Coupled Magnetics Design Tool," Available: http://www.princeton.edu/ $\sim$ minjie/coupL/coupL.html. 